\title{
New Concepts for Improved Durability of MOM Total Hip Endoprostheses. A Review
}

\author{
Lucian Capitanu, ${ }^{1, *}$, Virgil Florescu ${ }^{2}$ \\ ${ }^{1}$ Tribology Departmen Institute of Solid Mechanics of the Romanian Academy, Bucharest, Postcode, 010141, Romania \\ ${ }^{2}$ Mechanical Departatment, Institute of Civil Engineering, Bucharest, Postcode, 050236, Romania
}

\begin{abstract}
Analyzing some hip prostheses retrieved by revision, the appearance of some forms of polishing of the femoral head were observed, as well as significant plastic deformations followed by local hardening. At the same time, obvious traces of wearing through fretting of the acetabular cup of UHMWPE, which in some studies are confounded or assimilated with the adhesive wear. Starting with the well-known fact that the rolling movement always has a lower friction compared to sliding friction, the authors have conceived and realized a pivoting movement joint on a "layer of balls" with "compensation space", placed between the acetabular cup and the femoral head. This technical MOM solution allows free self-directed migration of the balls, depending on the resistance opposed, with successive occupation of the "compensation space". As a concept, the proposed technical solution excludes the existence of a cage for maintaining the spheres relative positions.
\end{abstract}

Keywords Total Hip Prosthesis, MOM, Self Directed Roliing Balls, Friction Coefficient, Stresses, Displacements

\section{Introduction}

This studies targeted contact mechanism including friction and wear phenomena accounting. The usual type of hip prostheses have high-density polyethylene cups (UHMWPE), ASTM-F648 (ISO 5834-2), and the femoral head from Stelit 21, ASTM-F 799 (ISO 5832-12). Based on the idea of reconsidering the potential of completely metallic type McKee-Farrar prosthesis, suggested by recent literature. The hip prosthesis have an acetabular cup made from Ti6Al4V ELI, ASTM-F 136 (ISO 5832-3/1990) alloy, with a density of de $4,5 \mathrm{~g} / \mathrm{cm}^{3}$, and the femoral head made from an CoCr-Mo alloy, with the density of de $8,8 \mathrm{~g} / \mathrm{cm}^{3}$, ASTM-F 799 (ISO 5832-12), was tested.

The proposed hip prosthesis, with self-directed rolling balls, was made using a femoral head from Stelit 21, the acetabular metallic cup from Ti6Al4V-ELI alloy and the balls from stainless steel. All the prostheses have the femoral head of $28 \mathrm{~mm}$ diameter. Theoretical and experimental researches done over the last 30 years have tried to contribute to the improvement of orthopedic endoprostheses durability, through constructive changes and even through changing their functional principle.

\section{Alternative Constructive Solutions}

* Corresponding author:

luciancapitanu@yahoo.com (Lucian Capitanu)

Published online at http://journal.sapub.org/materials

Copyright (C) 2012 Scientific \& Academic Publishing. All Rights Reserved

\subsection{Hip Endoprosthesis with Wheels}

Total hip endoprostheses with wheels represent one of the first attempts to decrease wear, by replacing the sliding movement with the rolling movement for one degree of freedom of the joint. This solution, suggested by a staff from the Imperial College of Science, Technology and Medicine (IC) in London, consists of the constructive modification of the modular hip prosthesis, by introducing a rolling bearing with conical wheels between the femoral stem neck and the femoral head[1]. The axis of the femoral stem neck represents the bearing's rotation axis. The surface of the neck represents one of the rolling paths, the other path being the surface of interior cavity in the femoral sphere. Since the rolling elements should be able to support a significant radial load in relation with the axial demand, the rotation axis of the femoral stem must satisfy the following conditions:

- it should coincide with the rotation axis of the femoral head in the acetabular cup, during most of the routine activities as normal walking

- it should be orthogonal to the the direction of resultant forces during normal walking.

It has been considered that a rolling bearing with conical wheels, that can support significant radial and axial loads, is most adequate for reaching this goal. But this requires a small diameter of the interior ring, imposed by the necessity of a reasonable diameter of the femoral head. Ensuring a satisfactory resistance for the bearing to challenge by fatigue is also necessary. Ensuring changeability of the femoral head is also imposed. Regarding these constraints, a cylindrical wheels (needles) bearing has been considered - Fig.1 (a). 
One must notice that rolling bearings with needles present a great durability to fatigue.

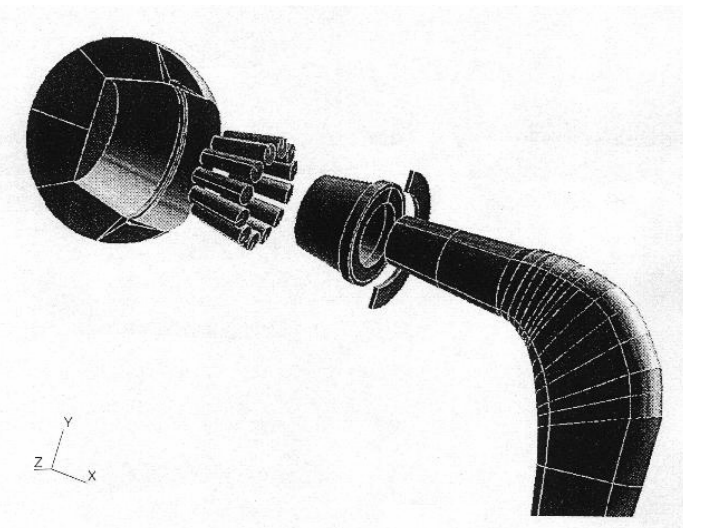

(a)

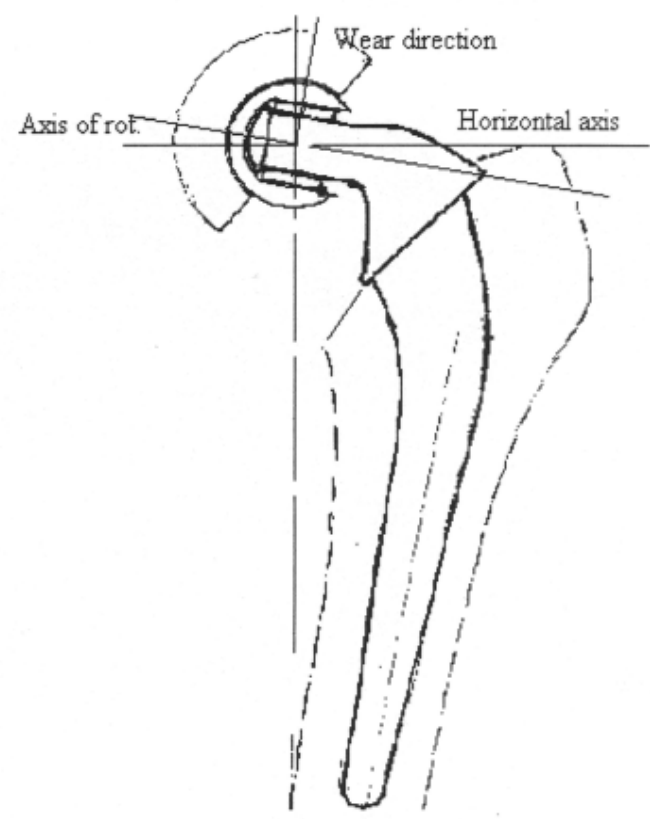

(b)

Figure 1. (a) Orthopaedic hip prosthesis with conical rolling elements around the femoral stem neck and (b) Total hip prosthesis with cylindrical wheels bearing[1]

Between the two variants, the one with rolling bearing with conical wheels was chosen due to its greater loading capacity, and to the fact that this kind of prosthesis can adapt to any combination of axial and radial forces. The configuration of the bearing with conical wheels also ensures a real rolling movement, throughout the contact length. It is regarded as a positive aspect the fact that the shape of bearing favors expulsion of foreign matters. Taking into account the simplicity of the assembling and disassembling procedures, an experimental model, shown in Fig.1 (b), has been realized and tested on a hip joint simulator. Since creating a rolling bearing with conical wheels of alloy $\mathrm{Co}-\mathrm{Cr}$ was not possible for this prototype, a Timken type bearing was used. The femoral head of the prototype was created from a stainless steel ball, with the diameter of $40 \mathrm{~mm}$, in order to ensure the necessary fit for assembling the conical wheels bearing. The method for establishing the quantitative aspects of wear was the gravimetric method, considering also the lubricant (physiological serum) absorption. The simulation was performed for 3 million loading cycles. After the first 500.000 cycles, oxidation of the femoral head was observed, manifested through color changes. The color of the interior acetabular cup's surface was also changed.

The superficial oxide layers were removed mostly by washing. The notable observed phenomenon was abrading the contact surface between the acetabular cup and the femoral head. Figures 2 (a) and 2 (b) show the weight modifications of the acetabular cup during the test.
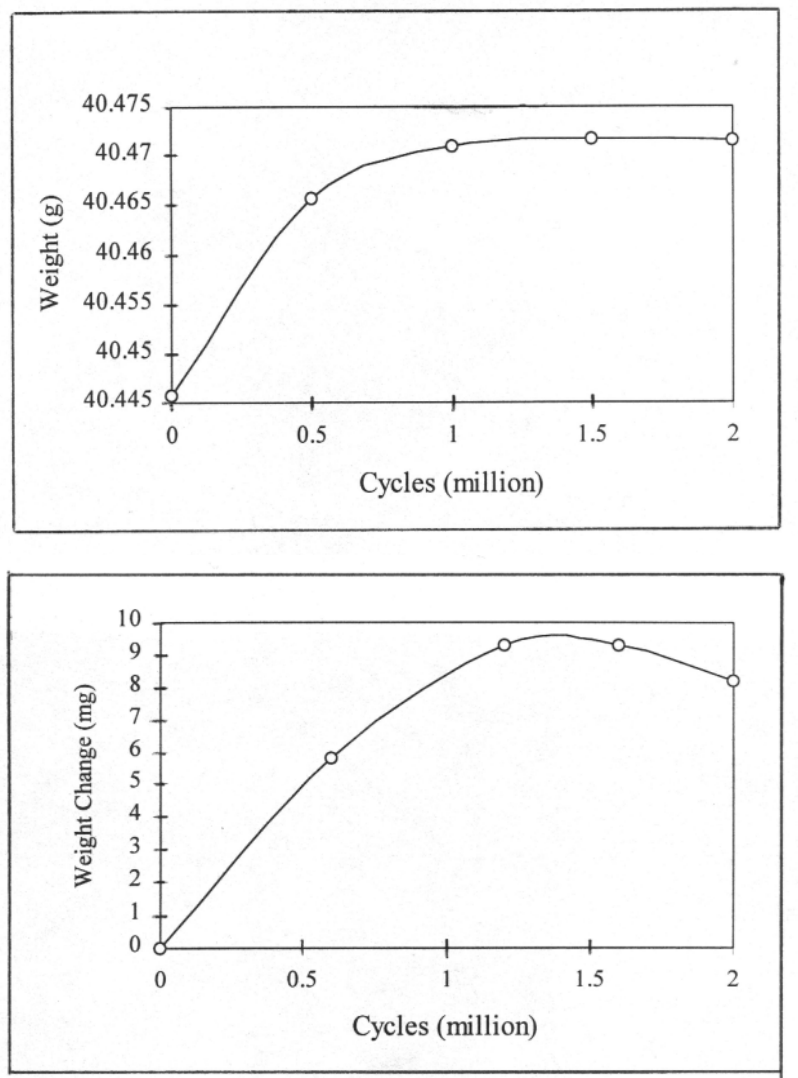

Figure 2. (a) Weight variation for the acetabular cup of the I.C. prototype during experimental tests and (b), weight modification for the acetabular's cup of the I.C. prototype after physiological serum absorption[1]

Unfortunately, the constructive solution proposed by the Imperial College researchers could not prevent the consequences of mechanical fatigue. This constructive solution leads to the failure of the prosthesis femoral neck stem after 2.3 millions cycles[1].

\subsection{Hip Endoprosthesis with Balls Train}

Other attempts regarding changing the constructive solution of total hip prosthesis took into consideration the fundamental change in the type of relative movement between the components of the total hip prosthesis. If, generally, the present technical solutions are based on the natural revolute movement of the femoral head in the acetabular cap, Katsutashi Bekki and Kiyoshi Shinjo[2] 
imagine a different design, the total hip prosthesis with „balls train”. This mainly consists of a dual joint construction (Fig. 3).
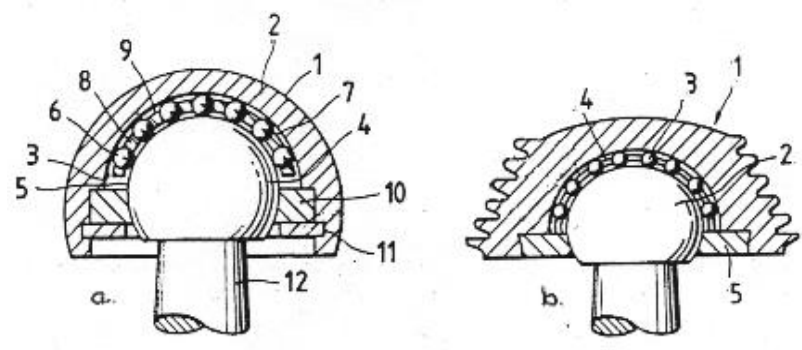

Figure 3. Sketch of the hip prosthesis with balls train[2]

The artificial joint is composed of an interior sphere (4) and an exterior cup (2) restricting the movement of a train of balls (7) mounted in a cage (8). By the characteristics of this design the movement is mainly by rolling. The interior cup surface (3) becomes the exterior collar of a bearing, the interior collar being the exterior surface (5) of the central sphere. The cage with the balls train is kept inside the working space by the screwed limiter (10), ensured against loosening by a blocking washer (11). Movement inside the joint implies point rolling contacts of the balls on the exterior and interior rolling path and a quasi-linear contact between the balls and their restrictive hole in the cage. The authors also propose constructive solutions for the press-fitting of the exterior cup in the acetabulum (Fig. 3a) or by screwing in it (Fig. 3b). In the proposed version, the central sphere and the rolling balls can be made from Co-Cr-Mo alloys, stainless steel or ceramic materials, and the exterior cup can be made from titanium or Cr-Mo alloys[2]. The cage and the fixation parts can be made out of biocompatible resins - high density polyethylene or polimethylmetacrylate[2]. Ceramic materials indicated by the authors include zirconium, siliceous nitrates, siliceous carbides and SiAlON compounds. The technical solution presented above allows the femoral head to rotate at $120^{\circ}$. The prosthesis design inventors claims that the friction coefficient in such prosthesis is appreciably equal to that of human natural joint.

\section{3. "Supertête" Prosthesis}

The French idea of the "Supertête" prosthesis[3] consists of placing the friction contact inside a bearing. The solution belongs to "Fundation de l'Avenir" in collaboration with "La Direction Générale de l'Armement (Ministère de la Défense, Mission Innovation)". In the opinion of French researchers, this technical solution can reduce the wear of the prosthesis with almost $99 \%$. In order to reach this result, a small spherical bearing of an "absolutely new" type, as the authors claimed, lubricated with synovial fluid, the natural lubricant of any prosthesis, was integrated in the femoral head (Fig. 4). Designed and built in accordance with aeronautics industry standards, the femoral head is claimed to have reduced wear, being designed to carry $25,000 \mathrm{~N}$, while the hip joint maximal forces do not overpass $5,000 \mathrm{~N}$. At this moment, the
„Supertête" prosthesis is a French Patent, an International Patent being under examination.

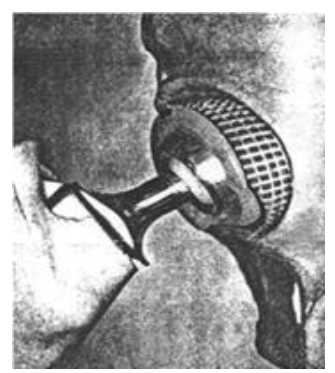

Figure 4. "Supertête" prosthesis[3]

Several prototypes, in different laboratory testing phases were made in order to establish the prosthesis' functioning period. The authors estimate that this artificial joint is able to function more than 30 years for a frequency of loading of approximately 1 million of cycles/year.

\subsection{MOM hip prosthesis with Self Directed Rolling Balls}

Another kind of total hip prosthesis with balls - Fig. 5, is proposed by the Institute of Solid Mechanics, of the Romanian Academy, in collaboration with the University of Medicine, and the University Hospital of Bucharest, making the object of a Romanian Patent[4].

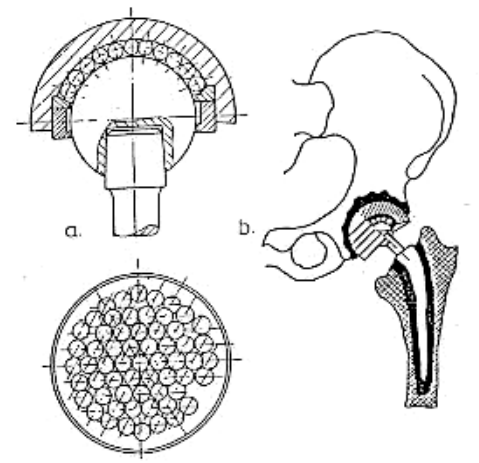

(a)

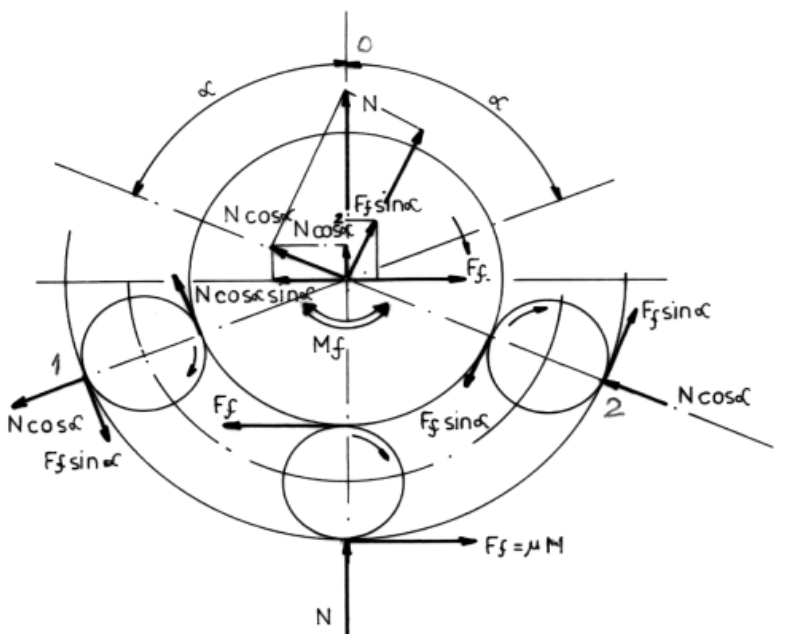

(b)

Figure 5. (a) Self-directed rolling balls scheme and (b) one of the fixation methods into the acetabulum, and the forces in transversal plane of proposed hip joint prosthesis[4] 
Analyzing some hip prostheses retrieved by revision, the appearance of some forms of polishing of the femoral head were observed, as well as significant plastic deformations followed by local hardening. At the same time, obvious traces of wearing through fretting of the acetabular cup of UHMWPE. In some studies this traces are confounded or assimilated with the adhesive wear[5-6]. Starting with the well-known fact that the rolling movement always has a lower friction compared to sliding friction, the authors have conceived and realized a pivoting movement joint on a "layer of balls" with "compensation space", placed between the acetabular cup and the femoral head. This technical solution allows free self-directed migration of the balls, depending on the resistance opposed, with successive occupation of the "compensation space" - Fig. 5(a). As a concept, the proposed technical solution excludes the existence of a cage for maintaining the spheres relative positions[7].

Figure 5(b) shows the forces equilibrium, after applying a normal load, at an oscillation angle to the vertical axis. As it can be seen, the normal force varies with the oscillation angle of the femoral head, in the same manner as the classical artificial articulation, but the magnitudes of the force and of the friction moment are decreased. Starting from these observations, a stand for testing artificial hip joints was constructed. Some comparative studies were realized on the usual hip prosthesis as well as on the complete metallic ones and on the new designed prosthesis with self-directed rolling balls. These studies targeted contact mechanism including friction and wear phenomena accounting. The usual type of hip prostheses have high-density polyethylene cups (UHMWPE), ASTM-F648 (ISO 5834-2), and the femoral head from Stelit 21, ASTM-F 799 (ISO 5832-12). Based on the idea of reconsidering the potential of completely metallic type McKee-Farrar prosthesis, suggested by recent literature, a total hip prosthesis having the acetabular cup made from Ti6A14V - ELI, ASTM-F 136 (ISO 5832-3/1990) alloy, with a density of de $4,5 \mathrm{~g} / \mathrm{cm}^{3}$, and the femoral head made from an Co-Cr-Mo alloy, with the density of de $8,8 \mathrm{~g} / \mathrm{cm}^{3}$, ASTM-F 799 (ISO 5832-12), was tested. The proposed hip prosthesis, with self-directed rolling balls, was made using a femoral head from Stelit 21, the acetabular metallic cup from Ti6Al4V -ELI alloy and the balls from stainless steel. All the prostheses have the femoral head of $28 \mathrm{~mm}$ diameter.

\section{Determination of the Position and the Maximum Number of Balls}

Practical realization of the joint described in Fig. 6(a) has proved that the free compensating space must be bigger than the volume of ball in order to avoid the blockage.

This studies have been carried out in order to determinate the possible positions and the maximum number of balls, with a given radius, that could be distributed in the rolling space. Studies are the pre-requisites of a subsequent, correct and complete, structural analysis[5-6]. The femoral head of the artificial joint being a sphere with radius $R$, the centers of the balls (having the radius $r$ ), tangent to this, are restrained to a spherical surface of $R+r$ radius (see Fig. 6.a).

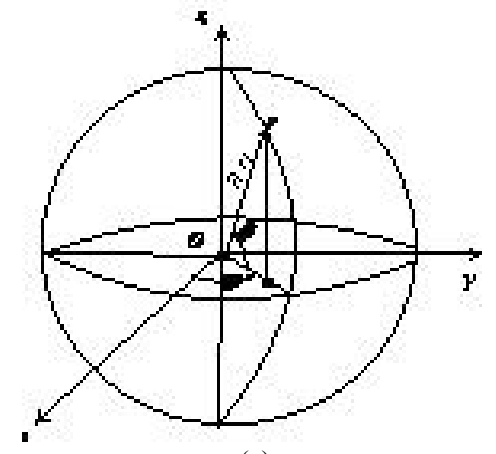

(a)

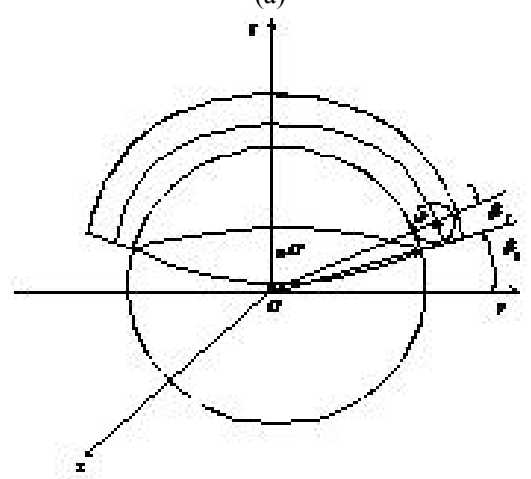

(b)

Figure 6. The position vector of a ball center tangent to the femoral head (a), and (b) the position of a ball in the rolling space[7]

If $P(x, y, z)$ is the center of a ball, the Cartesian coordinates will be:

$$
\begin{gathered}
x=(R+r) \cos \beta \cos \varphi, \\
y=(R+r) \cos \beta \sin \varphi, \\
z=(R+r) \sin \beta, \text { where } \beta \in(\beta \mathrm{s}, \pi / 2), \varphi \in(0,2 \pi) .
\end{gathered}
$$

The distance between the $P_{i}\left(x_{i}, y_{i}, z_{i}\right)$ and $P_{j}\left(x_{j}, y_{j}, z_{j}\right)$ centers, of any two spheres is calculated in the formula:

$$
\begin{gathered}
d_{i j}{ }^{2}=\left|P_{i} P_{j}\right|^{2}= \\
2(R+r)^{2}\left[1-\sin \beta_{i} \sin \beta_{j}-\cos \beta_{i} \cos \beta_{j} \cos \left(\varphi_{i}-\varphi_{j}\right)\right]
\end{gathered}
$$

Under the action of their own weight, the balls are arranged on rows, so that any ball in a row, except for the first, is simultaneously tangent to two consecutive balls from the inferior row.

The distance between the $P_{i}\left(x_{i}, y_{i}, z_{i}\right)$ şi $P_{j}\left(x_{j}, y_{j}, z_{j}\right)$, centers of two adjacent spheres, belonging to a row, must satisfy the condition:

$$
2 \mathrm{r} \leq \mathrm{d}_{\mathrm{ij}}<4 \mathrm{r}
$$

The first row consists of balls tangent to the rolling space basis, with centers situated on the parallel circle $\beta=\beta_{s}+\beta_{1}$ (see Fig. 6b), so that:

$$
\begin{gathered}
\mathrm{x}=(\mathrm{R}+\mathrm{r}) \cos (\beta \mathrm{s}+\beta 1) \cos \varphi \\
y=(R+r) \cos \left(\beta_{\mathrm{s}}+\beta_{1}\right) \sin \\
\mathrm{z}=(\mathrm{R}+\mathrm{r}) \sin (\beta \mathrm{s}+\beta 1)
\end{gathered}
$$

where:

$$
\beta_{1}=\arcsin \left(\frac{r}{R+r}\right)
$$


For two tangent balls, we have:

$$
\beta_{i}=\beta_{j}=\beta_{\mathrm{s}}+\beta_{1} ; d_{i j}=2 r
$$

As a result of (2), we have:

$$
2 r^{2}=(R+r)^{2} \cos ^{2}\left(\beta_{s}+\beta_{1}\right)\left[1-\cos \left(\varphi_{i}-\varphi_{j}\right)\right]
$$

The $\Delta \varphi$ angle between the centers of two tangent spheres can be determined from (7).

$$
\Delta \varphi=\varphi_{i}-\varphi_{j} \arccos \left(1-\frac{2 r^{2}}{(R+r)^{2} \cos ^{2}\left(\beta_{s}+\beta_{1}\right)}\right)
$$

The maximum number of tangent balls situated on the first row, having their centers situated on the parallel circle $\beta=\beta_{\mathrm{s}}$ $+\beta_{1}$ will be:

$$
\Delta \varphi=\varphi_{i}-\varphi_{j}=\arccos \left(1-\frac{2 r^{2}}{(R+r)^{2} \cos ^{2}\left(\beta_{s}+\beta_{1}\right)}\right)(9)
$$

In relation (9), $2 \pi / \Delta \phi$ represents the integer part of $2 \pi / \Delta \phi$.

The $\left(\varphi_{k}, \beta_{k}\right)$ coordinates of the $P_{k}$ centers, corresponding to the balls situated on the first row are:

$\phi_{k}=(k-1) \Delta \phi ; \beta_{k}=\beta_{s}+\beta_{1}$, where: $\left(k=1, \mathrm{n}_{0}\right) \quad(10)$

After determining the arrangement of the balls on the first row, the coordinates of the centers of the balls situated on the next row must be established. It is assumed that each ball from this row will be tangent to two other balls from the first row - Fig.7 (a) and (b). Generally speaking, the positions of the $P_{j}$ and $P_{k}$ centers of two balls, that satisfy the (3) conditions, are assumed to be known.

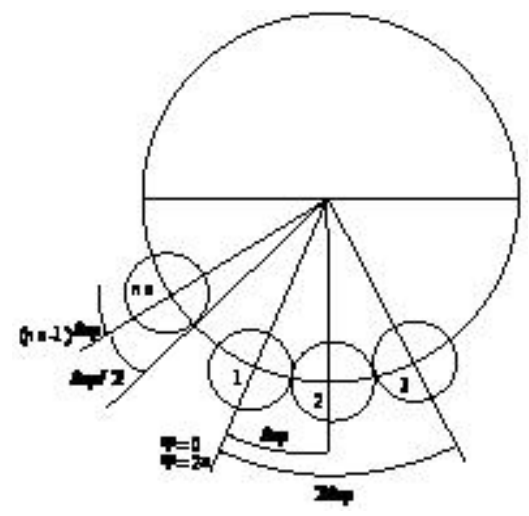

(a)

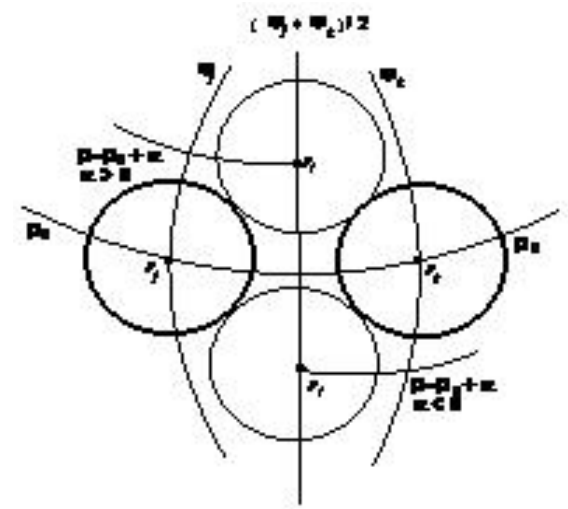

(b)

Figure 7. (a) Setting of the balls on the first row, and (b) tangent $P_{i}$ balls, when $\beta_{\mathrm{j}}=\beta_{\mathrm{k}[}$ ] $]$
Under these hypotheses, it has been proved that there are two balls tangent, up and down, to the balls from the first row. The following conditions are imposed:

$$
d_{i j}=d_{i k}=2 r \text { and } d_{i j}^{2}=d_{i k}^{2}=4 r^{2}
$$

Since the initial balls are on the $\beta=\beta_{0}$ parallel circle, we have $\beta_{j}=\beta_{k}=\beta_{0}$, and the equation system (11), for determining the $\left(\beta_{i}, \varphi_{i}\right)$ angles, can be written in the following form:

$$
\begin{aligned}
& 1-\sin \beta_{i} \sin \beta_{0}-\cos \beta_{i} \cos \beta_{0} \cos \left(\varphi_{i}-\varphi_{j}\right)=\frac{2 r^{2}}{(R+r)^{2}} \\
& 1-\sin \beta_{i} \sin \beta_{0}-\cos \beta_{i} \cos \beta_{0} \cos \left(\varphi_{i}-\varphi_{k}\right)=\frac{2 r^{2}}{(R+r)^{2}}
\end{aligned}
$$

From (12), we obtain:

$$
\cos \left(\varphi_{i}-\varphi_{j}\right)=\cos \left(\varphi_{k}-\varphi_{i}\right) \Rightarrow \varphi_{i}=\frac{\varphi_{k}+\varphi_{j}}{2}
$$

By replacing the (13) value for $\varphi_{i}$, in (12) we get:

$$
\sin \beta_{i} \sin \beta_{0}+\cos \beta_{i} \cos \beta_{0} \cos \left(\frac{\varphi_{k}-\varphi_{j}}{2}\right)=1-\frac{2 r^{2}}{(R+r)^{2}}
$$

In order to underline the differences between the balls that are tangent in the upper side, respectively lower side, the following relation is introduced:

$$
\beta_{\mathrm{i}}=\beta_{0}+\alpha
$$

In Fig. 7 it can be seen that $\alpha>0$, for the upper tangent balls and $\alpha<0$, for the lower tangent balls. The (14) equation becomes:

$$
\begin{aligned}
& \left(\sin \beta_{0} \cos \alpha+\cos \beta_{0} \sin \alpha\right) \sin \beta_{0}+ \\
& +\left(\cos \beta_{0} \cos \alpha+\sin \beta_{0} \sin \alpha\right) \cos \beta_{0} \cos \frac{\varphi_{1}-\varphi_{j}}{2}=c
\end{aligned}
$$

where:

$$
c=1-\frac{2 r^{2}}{(R+r)^{2}}
$$

By introducing the notations:

$$
\begin{aligned}
& a=\sin ^{2} \beta_{0}+\cos ^{2} \beta_{0} \cos \frac{\varphi_{1}-\varphi_{j}}{2} \\
& b=\sin \beta_{0} \cos \beta_{0}\left(1-\cos \frac{\varphi_{1}-\varphi_{j}}{2}\right)
\end{aligned}
$$

the $\alpha$ angle satisfies the equation:

$$
\phi_{k}=(k-1) \Delta \phi ; \beta_{k}=\beta_{s}+\beta_{l} \text {, where: }\left(k=1, \mathrm{n}_{0}\right)
$$

This equation can also be written like:

$$
(a+c) \operatorname{tg}^{2} \frac{\alpha}{2}-2 b \operatorname{tg} \frac{\alpha}{2}+c-a=0
$$

Solutions for the (18) equation are:

$$
\operatorname{tg} \frac{\alpha}{2}=\frac{b^{2} \pm \sqrt{b^{2}+a^{2}-c^{2}}}{a+c}
$$

For the upper tangent sphere, we have :

$$
\beta_{i}=\beta_{0}+2 \operatorname{arctg}\left(\frac{b^{2}+\sqrt{b^{2}+a^{2}-c^{2}}}{a+c}\right)
$$

Relations (19) and (13) show that, from a mathematical point of view, starting from any pair of adjacent balls from the first row, there is an upper tangent ball, to these. This means that the second row of balls „can consist” of the same 
number of balls as the first one. From a physical point of view, the distance between the centers of two adjacent balls must satisfy the (3) conditions.

Since every ball from the first row, except the $\left(n_{0}\right)$ and (1) balls, are tangent, the adjoining balls obtained on the second row would have the distances between centers smaller than $2 r$. That is why the number of balls from the second row will be smaller than that of the balls from the first row. This number can be calculated based on this relation:

$$
n_{1}=\left[n_{0} / 2\right]+1
$$

Next, we shall note with ,i" the ball that has $P_{i}$ as a center and $\left(\beta_{j i} ; \varphi_{i}\right)$ as angles. The iterative method, through which the balls belonging to the second row are obtained, consists of the following:

The tangent ball, noted $\left(n_{0}+1\right)$, is obtained from the $\left(n_{0}\right.$, 1) pair,

The ball $\left(n_{0}+2\right)$ is obtained from the pair $(2,3)$,

The ball $\left(n_{0}+3\right)$ is obtained from the pair $(4,5)$

The ball $\left(n_{i}-1\right)$ is obtained from the pair $\left(n_{0}-3, n_{0}-2\right)$.

The ball number $\left(n_{i}\right)$ can be obtained as tangent to balls $\left(n_{0}\right.$ $\left.-1, n_{0}\right)$, that have their centers situated on the same parallel circle.

Next, the solution to such a situation is presented.

We consider the $\left(P_{j}, P_{k}\right)$ balls, situated on different parallel circles, so that $\beta_{j} \neq \beta_{k}$ and we suppose $\varphi_{k}>\varphi_{j}$.

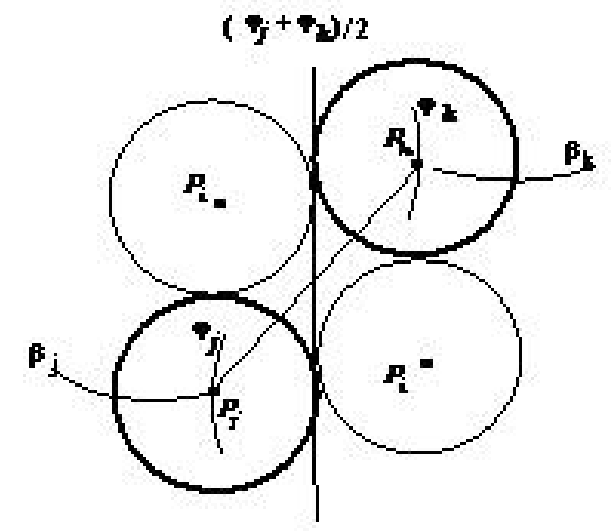

(a)

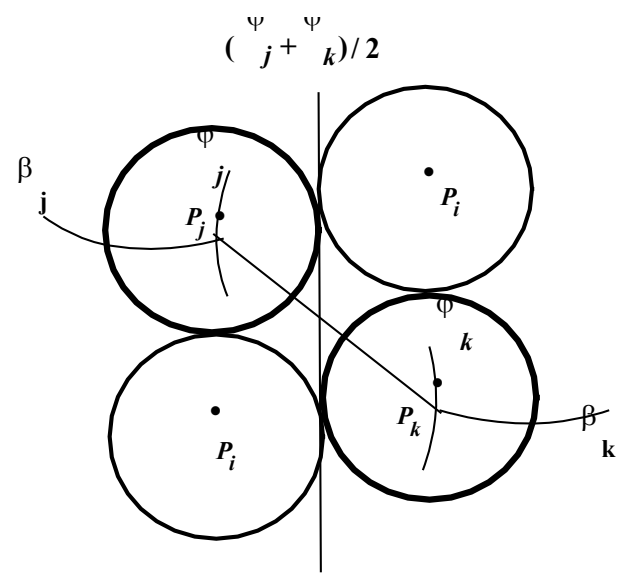

(b)

Figure 8. (a) Tangent $P_{i}$ balls for $\beta_{j}>\beta_{k}$. and (b) tangent $P_{i}$ balls for $\beta$ $<\beta_{k}$. [8]
In order to determine the $\left(\varphi_{i}, \beta_{i}\right)$ angles of the $P_{i}$ balls, tangent to $P_{j}$ and $P_{k}$, we will use the notation:

$$
\varphi_{i}=\frac{\varphi_{j}+\varphi_{k}}{2}+\varphi_{0}
$$

For $\beta_{\mathrm{j}}>\beta_{\mathrm{k}}$ it can be seen, from Fig. 8a, that: $\varphi_{0}<0$ - for the upper tangent ball and $\varphi_{0}>0$ - for the lower tangent ball.

In the second case, where $\beta_{\mathrm{j}}<\beta_{\mathrm{k}}$ (Fig. 8.b), it can be seen that: $\varphi_{0}>0$ - for the upper tangent ball and $\varphi_{0}<0$ - for the lower tangent ball.

The system of equations for determining the $\left(\beta_{i}, \varphi_{i}\right)$ angles can be obtained using (3) and has the following form:

$$
\begin{aligned}
& 2(R+r)^{2}\left[1-\sin \beta_{\mathrm{i}} \sin \beta_{\mathrm{j}}-\cos \beta_{\mathrm{i}} \cos \beta_{\mathrm{j}} \cos \left(\varphi_{\mathrm{i}}-\varphi_{\mathrm{j}}\right)\right]=4 r^{2} \\
& 2(R+r)^{2}\left[1-\sin \beta_{\mathrm{i}} \sin \beta_{\mathrm{k}}-\cos \beta_{\mathrm{i}} \cos \beta_{\mathrm{k}} \cos \left(\varphi_{\mathrm{i}}-\varphi_{\mathrm{k}}\right)\right]=4 r^{2}
\end{aligned}
$$

Using the (16) and (21) notations, equations (22) are written as follows:

$$
\begin{gathered}
\sin \beta_{\mathrm{i}} \sin \beta_{\mathrm{j}}+\cos \beta_{\mathrm{i}} \cos \beta_{\mathrm{j}} \cos \left(\varphi_{0}+\frac{\varphi_{\mathrm{k}}-\varphi_{\mathrm{j}}}{2}\right)=c \\
\sin \beta_{\mathrm{i}} \sin \beta_{\mathrm{k}}+\cos \beta_{\mathrm{i}} \cos \beta_{\mathrm{k}} \cos \left(\varphi_{0}-\frac{\varphi_{\mathrm{k}}-\varphi_{\mathrm{j}}}{2}\right)=c
\end{gathered}
$$

By introducing the notation:

$$
\alpha=\frac{\varphi_{\mathrm{k}}-\varphi_{\mathrm{j}}}{2}
$$

the (23) system of equations, for the $\left(\beta_{i} ; \varphi_{0}\right)$ quantities, becomes:

$$
\begin{aligned}
& \sin \beta_{\mathrm{i}} \sin \beta_{\mathrm{j}}+\cos \beta_{\mathrm{i}} \cos \beta_{\mathrm{j}}\left(\cos \varphi_{0} \cos \alpha-\sin \varphi_{0} \sin \alpha\right)=c \\
& \sin \beta_{\mathrm{i}} \sin \beta_{\mathrm{k}}+\cos \beta_{\mathrm{i}} \cos \beta_{\mathrm{k}}\left(\cos \varphi_{0} \cos \alpha+\sin \varphi_{0} \sin \alpha\right)=c
\end{aligned}
$$

By subtracting equation (25) from equation (25.1) we get: $\sin \beta_{i}\left(\sin \beta_{j}-\sin \beta_{k}\right)=\cos \beta_{i}\left[\cos \varphi_{0} \cos \alpha\left(\cos \beta_{k}-\cos \beta_{i}\right)(26)\right.$ $\left.+\sin \varphi_{0} \sin \alpha\left(\cos \beta_{j}+\cos \beta_{k}\right)\right]$

Since $\beta_{j} \neq \beta_{k}$, from (26) results:

$$
\operatorname{tg} \beta_{\mathrm{i}}=A \cos \varphi_{0}+B \sin \varphi_{0}
$$

In (27) the following notations were used:

$$
\begin{aligned}
& A=\cos \alpha\left(\cos \beta_{\mathrm{k}}-\cos \beta_{\mathrm{j}}\right) /\left(\sin \beta_{\mathrm{j}}-\sin \beta_{\mathrm{k}}\right) \\
& B=\sin \alpha\left(\cos \beta_{k}+\cos \beta_{j}\right) /\left(\sin \beta_{j}-\sin \beta_{k}\right)
\end{aligned}
$$

Now, an equation for determining the $\varphi_{0}$ angle can be obtained by using the formulas:

$$
\sin \beta_{\mathrm{i}}=\frac{\operatorname{tg} \beta_{\mathrm{i}}}{\sqrt{1+\operatorname{tg}^{2} \beta_{\mathrm{i}}}} \cos \beta_{\mathrm{i}}=\frac{1}{\sqrt{1+\operatorname{tg}^{2} \beta_{\mathrm{i}}}}
$$

For example, from (25.1) we get: $\sin \beta_{\mathrm{j}} \operatorname{tg} \beta_{\mathrm{i}}+\cos \beta_{\mathrm{j}}\left(\cos \varphi_{0} \cos \alpha-\sin \varphi_{0} \sin \alpha\right)$

$$
=c \sqrt{1+\operatorname{tg}^{2} \beta_{\mathrm{i}}}
$$

Using (27) we get:

$$
\begin{aligned}
\cos \varphi_{0}\left(A \sin \beta_{\mathrm{j}}\right. & +\cos \alpha \cos \beta)+\sin \varphi_{0}\left(B \sin \beta_{\mathrm{j}}-\sin \alpha \cos \beta_{\mathrm{j}}\right) \\
= & c \sqrt{1+\left(A \cos \varphi_{0}+B \sin \varphi_{0}\right)^{2}}
\end{aligned}
$$

This equation can be written as:

$$
\begin{aligned}
& \mathrm{A}_{1} \cos \varphi_{0}+\mathrm{B}_{1} \sin \varphi_{0}= \\
& =c \sqrt{1+\mathrm{A}^{2} \cos ^{2} \varphi_{0}+\mathrm{B}^{2} \sin ^{2} \varphi_{0}+\mathrm{AB} \sin 2 \varphi_{0}}
\end{aligned}
$$

where:

$A_{1}=A \sin \beta_{1}+\cos \alpha \cos \beta_{\mathrm{j}} ; B_{1}=B \sin \beta_{\mathrm{j}}-\sin \alpha \cos \beta_{\mathrm{j}}(33)$ 
Another form for (32) is:

$$
\begin{aligned}
& \cos ^{2} \varphi_{0}\left(\mathrm{~A}_{1}^{2}-\mathrm{A}^{2} \mathrm{c}^{2}\right)+\sin ^{2} \varphi_{0}\left(\mathrm{~B}_{1}^{2}-\mathrm{B}^{2} \mathrm{c}^{2}\right) \\
& +\sin 2 \varphi_{0}\left(\mathrm{~A}_{1} \mathrm{~B}_{1}-\mathrm{AB} c^{2}\right)=\mathrm{c}^{2}
\end{aligned}
$$

This equation for calculating the $\varphi_{0}$ angle can be written as:

where:

$$
A_{2} \cos 2 \varphi_{0}+B_{2} \sin 2 \varphi_{0}=C_{2}
$$

$$
\begin{gathered}
A_{2}=A_{1}^{2}-B_{1}^{2}+\left(B^{2}-A^{2}\right) c^{2} \\
B_{2}=2\left(A_{1} B_{1}-A B c^{2}\right) \\
C_{2}=-A_{1}^{2}+B_{1}^{2}+\left(A^{2}-B^{2}\right) c^{2}
\end{gathered}
$$

Equation (35) is similar to (17), so it can be written as:

$$
\left(C_{2}+A_{2}\right) \operatorname{tg}^{2} \varphi_{0}-2 B_{2} \operatorname{tg} \varphi_{0}+C_{2}-A_{2}=0 \text { (37) }
$$

The solutions to the equation are:

$$
\operatorname{tg} \varphi_{0}=\frac{B_{2} \pm \sqrt{B_{2}^{2}+A_{2}^{2}-C_{2}^{2}}}{A_{2}+C_{2}}
$$

For the upper tangent sphere, when $\beta_{j}>\beta_{k}$ we have:

$$
\varphi_{0}=\operatorname{arctg}\left(\frac{B_{2}+\sqrt{B_{2}^{2}+A_{2}^{2}-C_{2}^{2}}}{A_{2}+C_{2}}\right)
$$

For $\beta_{j}<\beta_{k}$, we get:

$$
\varphi_{0}=\operatorname{arctg}\left(\frac{B_{2}-\sqrt{B_{2}^{2}+A_{2}^{2}-C_{2}^{2}}}{A_{2}+C_{2}}\right)
$$

Now, from (27) and (21) we can get:

$$
\beta_{\mathrm{i}}=\operatorname{arctg}\left(A \cos \varphi_{0}+B \sin \varphi_{0}\right) \varphi_{\mathrm{i}}=\frac{\varphi_{\mathrm{j}}+\varphi_{\mathrm{k}}}{2}+\varphi_{0}(
$$

Based on the obtained theoretical results, two programs FORTRAN were built for determining balls position in the rolling space and their graphic representation.

Under the action of their own weight, the balls from the rolling space arrange themselves on overlapped rows. The first row consists of balls that are simultaneously tangent to the sphere and to the rolling surface and, therefore, their centers are on a parallel circle.

Two consecutive balls, $(i)$ and $(i+1)$, are tangent to each other, so that

$$
d_{i, i+1}=2 r
$$

The angle between two consecutive balls, noted $\Delta \varphi$, is determined from the (42) condition, with the (8) relation.

The number of balls situated on the first row was noted $n_{0}$ and was determined by the (9) relation. Due to the rotation symmetry it can be assumed, without diminishing the degree of generality, that the first ball is on the first meridian, that is $\varphi_{1}=0,\left(\varphi_{k}, \beta_{k}\right)$ are calculated with (10). In order to obtain the other rows of spheres, it has been proved that, for any $(j)-(k)$ pair of balls, satisfying the conditions:

$$
2 r<d_{j k}<4 r, \varphi_{j}<\varphi_{k}
$$

there is an $(i)$ ball, upper tangent to the first two.

We distinguish three cases, depending on the $\beta_{j}$ şi $\beta_{k}$ values.
Case I, $\beta_{j}=\beta_{k}=\beta_{0}$ results:

$\varphi_{i}=\left(\varphi_{j}+\varphi_{k}\right) / 2$

$$
\beta_{\mathrm{i}}=\beta_{0}+2 \operatorname{arctg}\left(\frac{b^{2}+\sqrt{b^{2}+a^{2}-c^{2}}}{a+c}\right)
$$

where $a, b, c$ are defined by (16) and (16) .

Case II, $\beta_{j}>\beta_{k}$;

Case III, $\beta_{j}<\beta_{k}$

In both last cases, the angles are calculated with (41) formulas, using (39) and (40) respectively. The second row has $n_{1}$ balls and the $\left(\varphi_{i}, \beta_{i}\right),\left(i=n_{0}+1, n_{0}+n_{1}-1\right)$ angles are calculated with (40).

Since the distance $d_{n_{0}+n_{1}-1, n_{0}+1}>4 r$, there is a ball noted by $\left(n_{0}+n_{1}\right)$, tangent to the $\left(n_{0}+n_{1}-1, n_{0}-1\right)$ pair of balls, or the $\left(n_{0}, n_{0}+1\right)$ pair. The $\left(\varphi_{\mathrm{n}_{0}}, \beta_{\mathrm{n}_{0}}\right)$ angles are determined by (41). The existence of the alternative mentioned above shows that the arrangement of the balls is not a unique construction. Starting from one row of balls, another row can be built out, consisting of balls tangent to pairs of balls of the starting row. A row is considered complete when the (43) condition is accomplished for any two consecutive balls from the respective row. The defining balls procedure is over when the $\beta_{k}$ angles (for the balls on the last row) have a value $\leq$ than $\pi / 2$. From the first FORTRAN program, made on the basis of the obtained results, one obtains a BL $(i, j),(i=$ $\left.1, N_{b}\right),(j=1,2)$ matrix, where:

- $N_{b}$ represents the total number of the balls,

- BL $(i, 1)$ is the $\beta_{i}$ angle of the ball number $(i)$,

- BL $(i, 2)$ is the $\varphi_{i}$ angle of the ball number $(i)$.

From the (1) relations, are obtained the Cartesian coordinates of the balls center in the rolling space, and as a consequence the final arrangements of the balls.

From the graphical part, realized with the second FORTRAN program, one obtains the ball positions representation, with the help of the BL $(i, j)$ matrix. The observer is placed on the $O^{\prime} x$ axis - see Fig. 6a. - in the first row's plane and in the direction of the first ball, so that $\varphi=0$. Images can be rotated around the $O^{\prime} z$ axis, when $\varphi \in(0,2 \pi)$, or around the $O^{\prime} y$ axis, when $\beta \in(-\pi / 2, \pi / 2)$. Numerical and graphical results are shown for an articulation with the following feature: $R=14 \mathrm{~mm}, r=1.25 \mathrm{~mm}, \beta=10^{\circ}$. In this case, a total number of $N_{b}=199$ spheres was obtained and the balls are arranged on 12 consecutive rows, numbered from $n_{0}$ to $n_{11}$ :

$n_{0}=37 ; n_{1}=19 ; n_{2}=19 ; n_{3}=19 ; n_{4}=19 ; n_{5}=19 ;$ $n_{6}=19 ; n_{7}=19 ; n_{8}=14 ; n_{9}=9 ; n_{10}=5 ; n_{11}=1$.

Images of the balls arrangement for $\varphi=0$ and $\beta=15^{0}$; $\beta=0^{0} ; \beta=-15^{0}$ are shown in Figs. 9 a - 9.c, respectively.

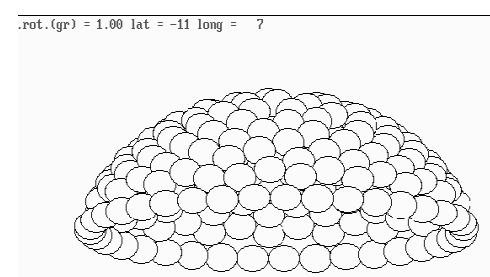




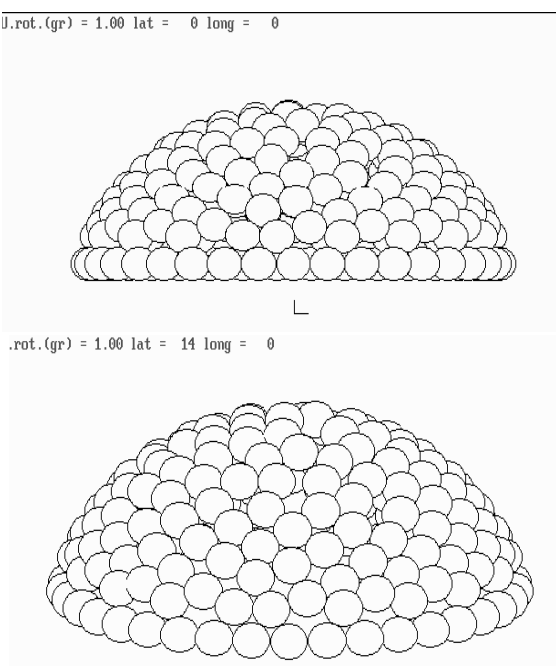

Figure 9. a. Lateral view switched up with $\beta=15^{\circ} ;$ b. Frontal view $(\beta=$ $\left.0^{0}\right)$; c. Lateral view switched down with $\beta=-15^{0[}[8]$

From the presented mathematical analysis, it results that the arrangement of the balls in the rolling space is asymmetrical and not uniquely determined.

\section{Experimental Studies}

Based on this studies shown, a self-directed rolling balls prosthesis was realized, having a femoral head diameter of $28 \mathrm{~mm}$ and 199 balls with a 2,5 $\mathrm{mm}$ diameter each. During the research we have obtained comparative measuring of the friction coefficient for three types of prosthesis, at $\pm 30^{\circ}$ oscillations, in the presence - or in the absence - of physiological serum used as lubricant. Fig. 10 shows a partial view of the drive system and the friction couple, femoral head / acetabular cup. The experimental device has been described in detail elsewhere.

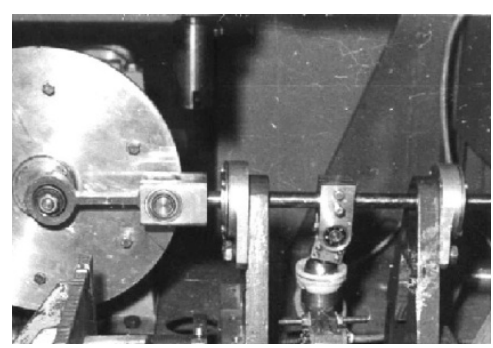

Figure 10. Partial view of drive system and the friction couple

For the experimental tries two comparison frequencies were used, namely 60 oscillations / min and 120 oscillations / min, corresponding to a normal and an accelerated step frequency.

The couples were submitted to normal loads demands of $500 \mathrm{~N}, 1000 \mathrm{~N}$ and $1500 \mathrm{~N}$. The values of the friction forces were measured, taking into account the variations of the normal force with the oscillation angle. Only the values of the friction forces in the (0) position of the couples (on the vertical direction) have been taken into consideration. These values are represented in Tabs. 1., 3., and 5 for dry friction conditions and in Tabs. 2, 4. and 6, for the physiological serum lubrication.

Table 1. Forces values and friction coefficients for Stelit 21 / UHMWPE in dry friction[7]

\begin{tabular}{|c|c|c|c|}
\hline$f($ osc $/$ min $)$ & $N(\mathrm{~N})$ & $F_{f}(\mathrm{~N})$ & $\mu$ \\
\hline 60 & 50 & 14.25 & 0.285 \\
\hline 60 & 100 & 32.00 & 0.320 \\
\hline 60 & 150 & 51.30 & 0.342 \\
\hline 120 & 50 & 14.00 & 0.280 \\
\hline 120 & 100 & 29.50 & 0.295 \\
\hline 120 & 150 & 45.00 & 0.300 \\
\hline
\end{tabular}

Table 2. Forces values and friction coefficients for Stelit 21 / UHMWPE in physiological serum[7]

\begin{tabular}{|c|c|c|c|}
\hline$f$ (osc/min) & $N(\mathrm{~N})$ & $F_{f}(\mathrm{~N})$ & $\mu$ \\
\hline 60 & 50 & 1.75 & 0.035 \\
\hline 60 & 100 & 4.90 & 0.049 \\
\hline 60 & 150 & 9.75 & 0.065 \\
\hline 120 & 50 & 1,60 & 0.032 \\
\hline 120 & 100 & 4.10 & 0.041 \\
\hline 120 & 150 & 6.75 & 0.045 \\
\hline
\end{tabular}

Table 3. Forces values and friction coefficients for CoCrMo alloy/Ti6Al4V in dry friction[7]

\begin{tabular}{|c|c|c|c|}
\hline$f(\mathrm{osc} / \mathrm{min})$ & $N(\mathrm{~N})$ & $F_{f}(\mathrm{~N})$ & $\mu$ \\
\hline 60 & 50 & 15.00 & 0.300 \\
\hline 60 & 100 & 31.20 & 0.312 \\
\hline 60 & 150 & 48.75 & 0.325 \\
\hline 120 & 50 & 15.75 & 0.315 \\
\hline 120 & 100 & 33.00 & 0.330 \\
\hline 120 & 150 & 51.75 & 0.345 \\
\hline
\end{tabular}

Table 4. Forces values and friction coefficients for CoCrMo alloy/Ti6A14V in physiological serum[7]

\begin{tabular}{|c|c|c|c|}
\hline$f(\mathrm{osc} / \mathrm{min})$ & $N(\mathrm{~N})$ & $F_{f}(\mathrm{~N})$ & $\mu$ \\
\hline 60 & 50 & 3.25 & 0.065 \\
\hline 60 & 100 & 7.30 & 0.073 \\
\hline 60 & 150 & 13.50 & 0.09 \\
\hline 120 & 50 & 4.250 & 0.085 \\
\hline 120 & 100 & 12.50 & 0.125 \\
\hline 120 & 150 & 21.15 & 0.141 \\
\hline
\end{tabular}

Table 5. Forces values and friction coefficients for self directed rolling sphere Stelit 21 / stainless steel / Ti6A14V - ELI, in dry conditions[7]

\begin{tabular}{|c|c|c|c|}
\hline$f(\mathrm{osc} / \mathrm{min})$ & $N(\mathrm{~N})$ & $F_{f}(\mathrm{~N})$ & $\mu$ \\
\hline 60 & 50 & 20.50 & 0.154 \\
\hline 60 & 100 & 35.25 & 0.205 \\
\hline 60 & 150 & 6.00 & 0.235 \\
\hline 120 & 50 & 15.80 & 0.120 \\
\hline 120 & 100 & 27.75 & 0.158 \\
\hline 120 & 150 & 27.75 & 0.185 \\
\hline
\end{tabular}

Table 6. Forces values and friction coefficients for self directed rolling sphere Stelit 21 / stainless steel / Ti6Al - ELI, in physiological serum[5]

\begin{tabular}{|c|c|c|c|}
\hline$f(\mathrm{osc} / \mathrm{min})$ & $N(\mathrm{~N})$ & $F_{f}(\mathrm{~N})$ & $\mu$ \\
\hline 60 & 50 & 0.30 & 0.006 \\
\hline 60 & 100 & 0.70 & 0.007 \\
\hline 60 & 150 & 1.20 & 0.008 \\
\hline 120 & 50 & 0.35 & 0.007 \\
\hline 120 & 100 & 0.80 & 0.008 \\
\hline 120 & 150 & 1.35 & 0.009 \\
\hline
\end{tabular}


According to this information, for most of the acetabular cups, the wear direction is between $10^{\circ}$ on the lateral and $10^{\circ}$ in the median direction. Therefore the region can be placed around the vertical axis of the acetabular cup, in an anatomical position, where it is located in live recordings. For some joints, the wear speeds can be influenced by abrasive particles turned up due to the deteriorating of the finished surface of the femoral head.

The variation domain of the friction coefficients, in the experimental conditions presented before, is presented in Table 7.

Table 7. Variation domain of the friction coefficients

\begin{tabular}{|c|c|}
\hline $\begin{array}{c}\text { couple type Stelit 21/UHMWPE, dry } \\
\text { friction }\end{array}$ & $\mu=0,285 \div 0,342$ \\
\hline $\begin{array}{c}\text { couple type Stelit } 21 / \text { UHMWPE, } \\
\text { lubricated }\end{array}$ & $\mu=0,035 \div 0,065$ \\
\hline $\begin{array}{c}\text {-Co-Cr-Mo / Ti6Al4V alloy cuple, dry } \\
\text { friction }\end{array}$ & $\mu=0,300 \div 0,345$ \\
\hline $\begin{array}{c}\text { - Co-Cr-Mo / Ti6Al4V alloy cuple } \\
\text { lubricated }\end{array}$ & $\mu=0,065 \div 0,141$ \\
\hline $\begin{array}{c}\text { - couple with balls in self-directed } \\
\text { movement, dry friction }\end{array}$ & $\mu=0,120 \div 0,205$ \\
\hline $\begin{array}{c}\text { - couple with balls in self-directed } \\
\text { movement, lubricated }\end{array}$ & $\mu=0,006 \div 0,009$ \\
\hline
\end{tabular}

It can be observed that the smallest values of the force and of the friction coefficient are obtained for the prostheses with balls and self-directed movement (approximately 5 times smaller than the values obtained on a classical prosthesis). For all the tested couples, the friction force grows at the growth of the normal load and of the oscillation speed.

The tests did not allow the quantitative measurement of the acetabular cups' wear and of the femoral head, as a consequence of the lacking of a comparative measurement method, adequate to all studied cuples. Still, some very clear qualitative observations were done, that enables the following conclusions:

- for the couple type Stelit $21 /$ UHMWPE, the wear is manifested through the uneven polished of the femoral head and through the appearance of some traces of wear by friction inside the polyethylene acetabular cup;

- Co-Cr-Mo / Ti6Al4V alloy couples, polishing and fine uneven scratches show up, on both elements of the prosthesis, even in the presence of physiological serum;

- the self-directed movement balls prosthesis did not show sings of wear. Under the testing conditions used in the laboratory, there were no traces of abrasion or seizing.

\section{Stresses and Displacements Analysis}

The tribological behaviour of the articulation with balls justifies a deeper analysis of the stresses and displacements under demanding conditions, compared to the classical articulation (Fig. 11).

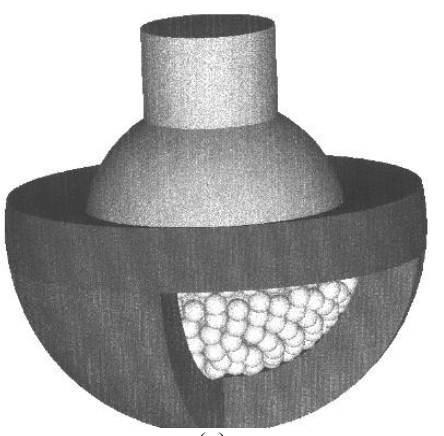

(a)

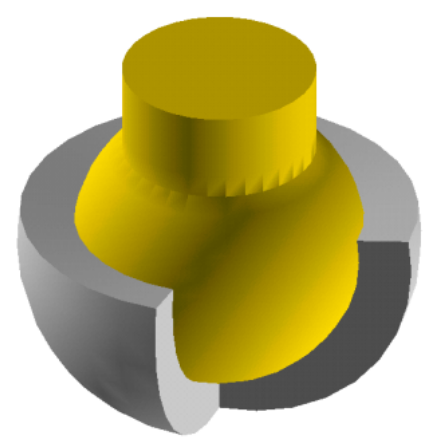

A USER INPUT

(b)

Figure 11. The three-dimensional simplified models: a- model with balls b- classical model[7]

This model considers the exterior surface of the acetabular cup built-in and the femoral head executes a rotation movement in the diametric plane, under the action of a compression force. As a result of the big number of contact points, the analysis was done for a very short duration of the movement (quasi-static analysis), of $0,8 \times 10^{-3} \mathrm{sec}$. It was established that the compression force and the rotation moment are to be constant throughout the demand period. In order to obtain the stresses and the displacements in the acetabular cup we suppose that the balls and the femoral head are rigid and that the acetabular cup is linearly elastic. The structures have been divided into finite elements of type SOLID 164-brick with 8 nodes. For the rigid parts a "constant stresses" formulation was used, and for the elastic one, "integrated totally", with the purpose of eliminating the „hourglass" nodes. The following mechanical characteristics, identical for the thighbone head, the spheres and the acetabular cap, were taken into account: Young's modulus: $E=2,1 \times 10^{11} \mathrm{~N} / \mathrm{m}^{2}$; density: $\rho=7850 \mathrm{~kg} / \mathrm{m}^{3}$; Poisson's coefficient: $v=0,3$.

A number of 58784 elements and 73100 nodes were taken into consideration.

In Figs. 12 a, b and c, networks of separation in finite elements, the femoral head, the balls and acetabular cup respectively, are presented. For finite element method (FEM) analysis software ANSYS was used. 


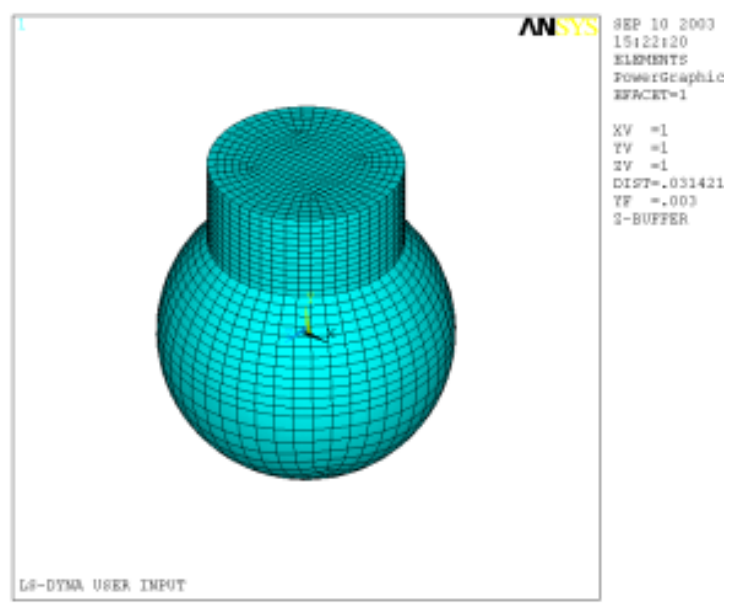

(a)

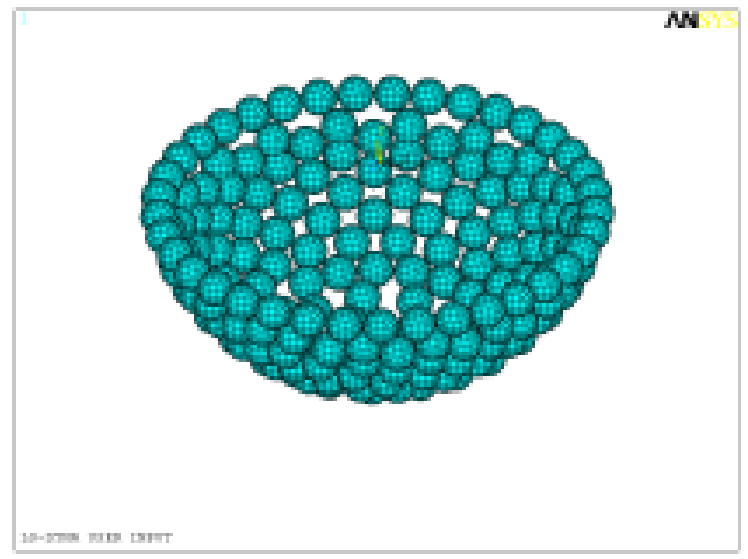

(b)

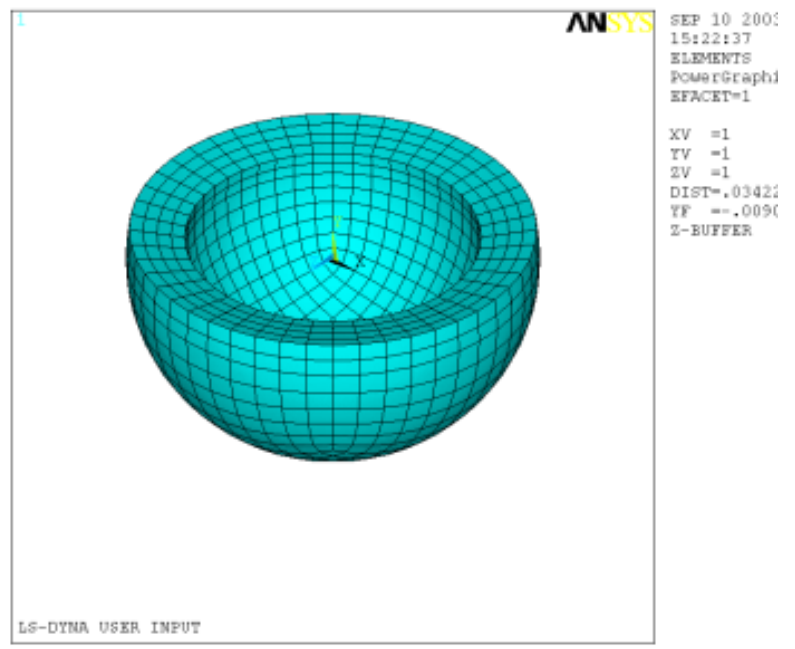

(c)

Figure 12. The finite elements discretisation for (a) the femoral head; (b) the finite elements of the balls and (c) the finite elements discretisatin of the acetabular cup[9]

It has been considered that on the $y$ direction operates a force of $3200 \mathrm{~N}$, approximately four times bigger than a man's medium weight.

The rotation moment, applied around the $z$ axis was of 44,8 Nm. Supposing that all the external surfaces are in contact, the value of the sliding friction coefficient was considered $v_{\mathrm{s}}=0,006$ and that of the rolling friction coefficient $v_{\mathrm{r}}=1,41 \times 10^{-4} \mathrm{~m}$.

The maximum tangential stress is calculated with:

$$
T_{\max }= \pm\left|\sigma_{1}-\sigma_{3}\right|
$$

where $\sigma_{1}, \sigma_{2}, \sigma_{3}$ are the main stresses and it was supposed that $\sigma_{1}<\sigma_{2}<\sigma_{3}$.

\section{Results and Disscusion}

In Figs. 13 and 14, the maximum tangential stresses and the normal displacements in the acetabular cup, are shown. They correspond to eight different instantaneous times, starting with $t_{1} \approx 0$ and $t_{8}=t_{\text {final }}$.
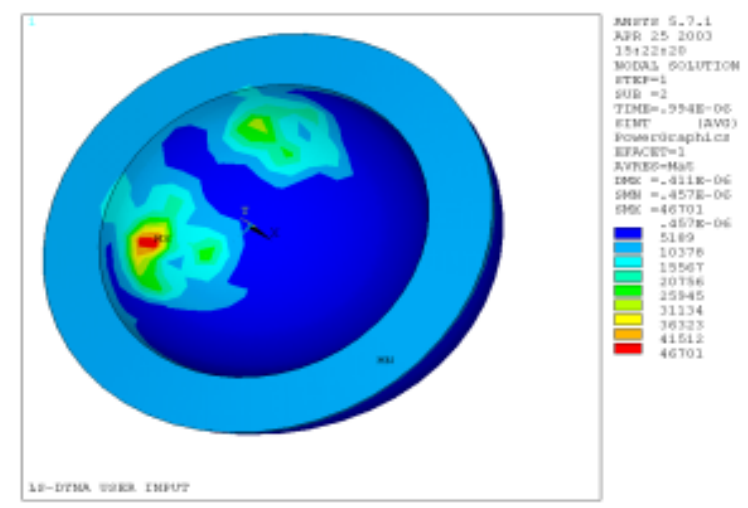

stresses 1

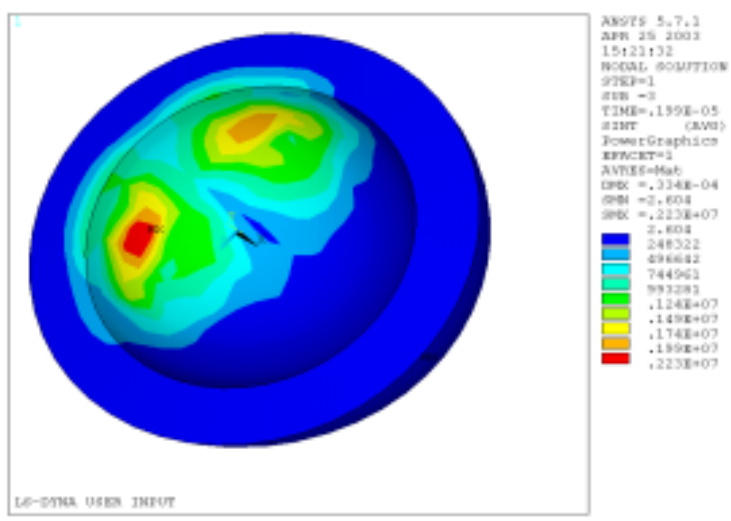

stresses 2
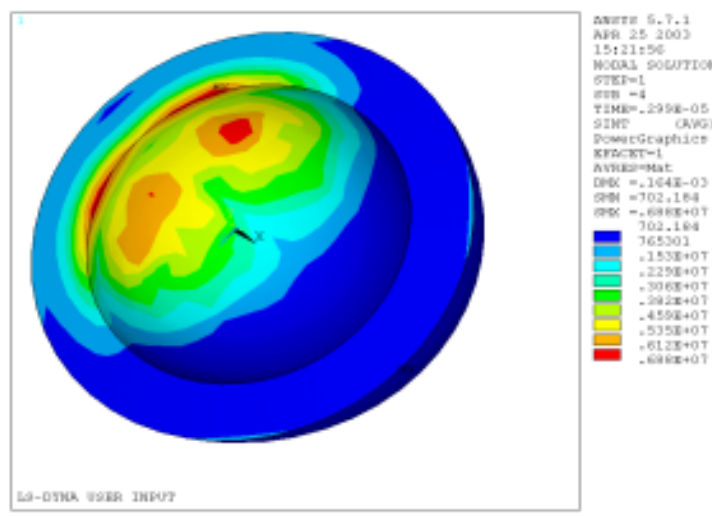

stresses 3 

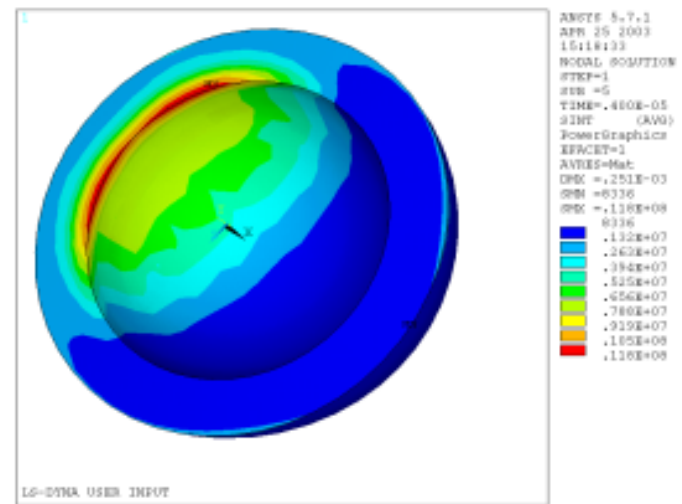

stresses 4

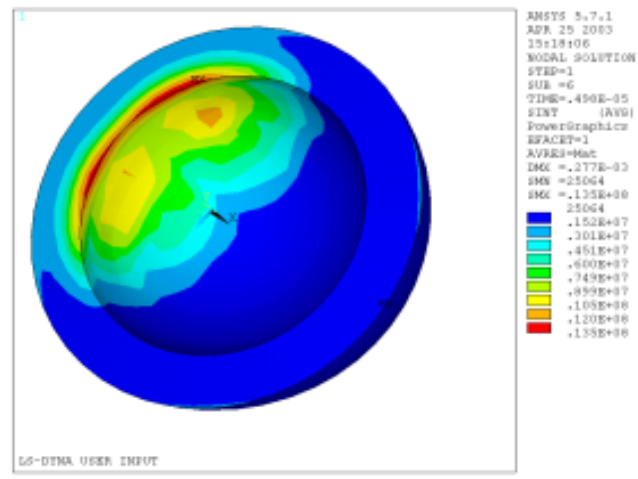

stresses 5

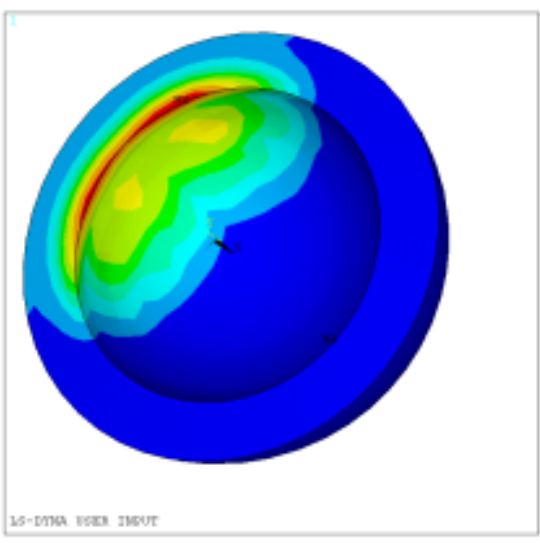

stresses 6

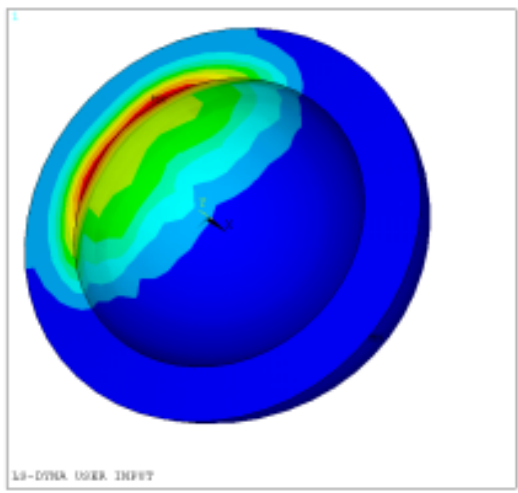

stresses 7

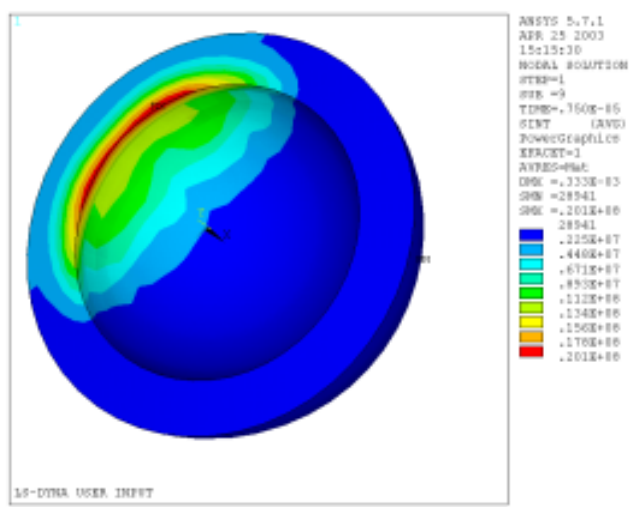

stresses 8

Figure 13. Maximal tangential stresses (in the acetabular cup) for eight different moments of the movement[9]

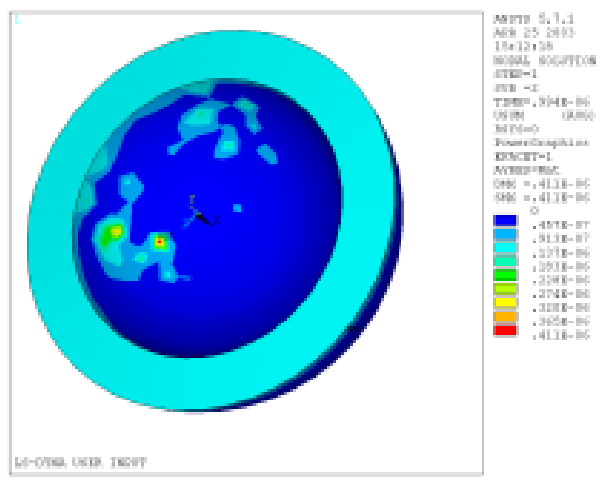

diplacements 1
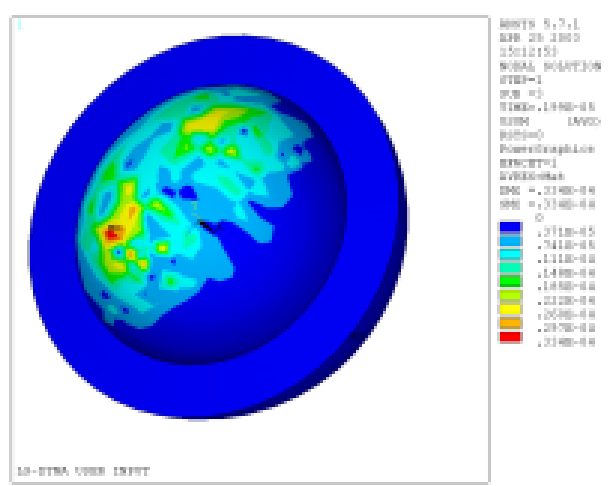

diplacements 2
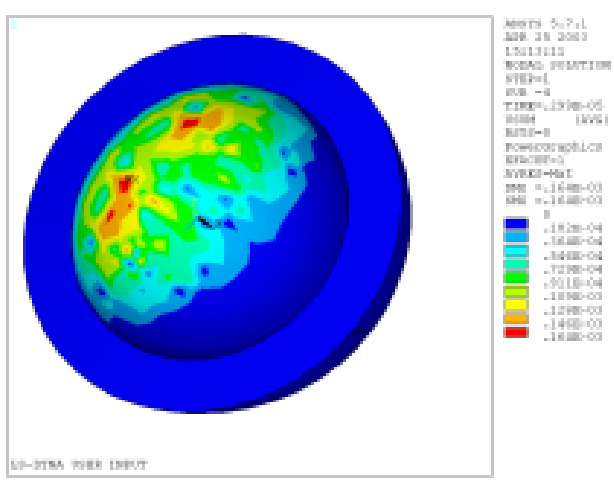

diplacements 3 

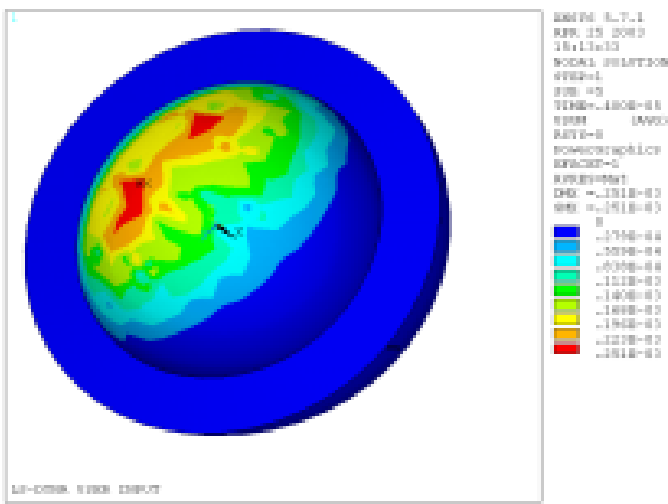

diplacements 4
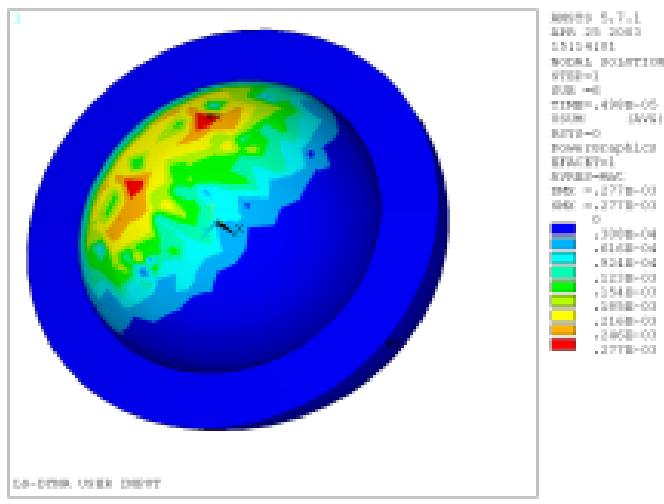

diplacements 5
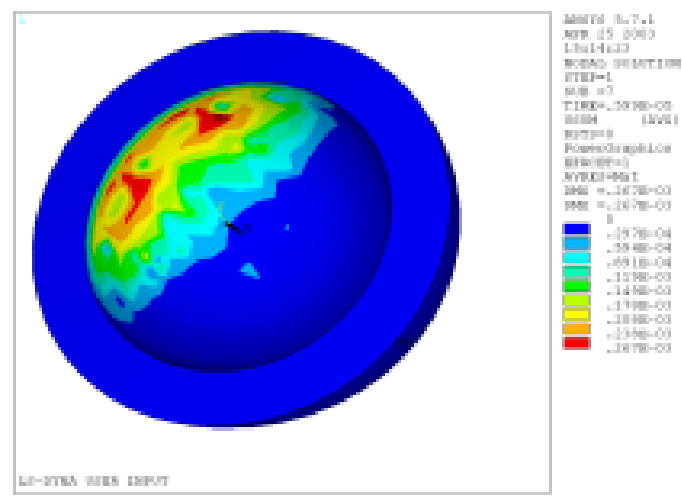

diplacements 6

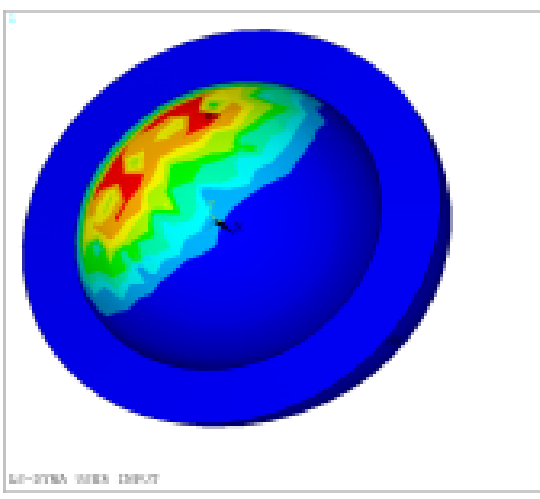

diplacements 7

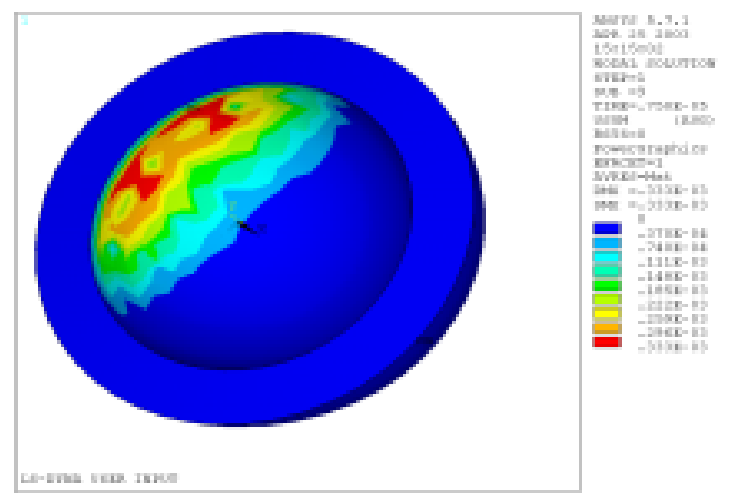

diplacements 8

Figure 14. Normal displacements (in the acetabular cup) during eight different moments of the movement[9]

Analyzing the recordings of the stresses and displacements in the acetabular cup, for the studied case, it results that the most requested balls are not on the movement direction, a fact otherwise predictable. They are found in the maximum resistance space produced in the escaping area (the blocking hoop).

Fig. 15 shows the variation of the energies during the deformation process.

The maximum value of the tangential stress, obtained for the last step of time, is very small compared to the admissible stress:

$$
T_{\max }=20 \mathrm{MPa}<<\sigma_{a}=1200 \mathrm{MPa}
$$

The maximum normal displacement was of $0,033 \mathrm{~mm}$. For a comparison, Figs 16 and respectively 17, show the variation of the maximum tangential stresses and respectively of the normal displacements of the metallic prosthesis, having the acetabular cup made out of Ti6Al4V-ELI alloy and the thighbone head out of Co-Cr-Mo alloy.

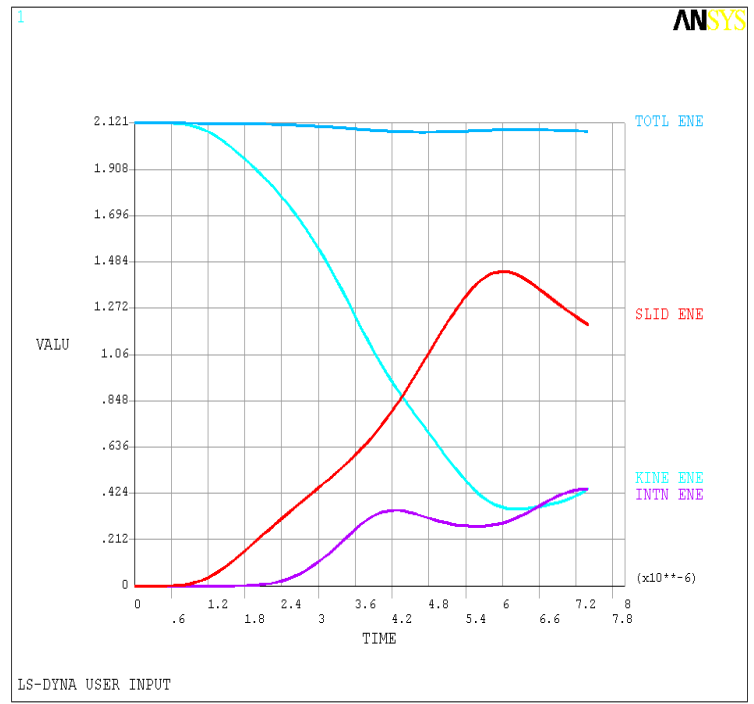

Figure 15. Energy variation diagram during the deformation process for the prosthesis with balls in self-directed movement[9] 


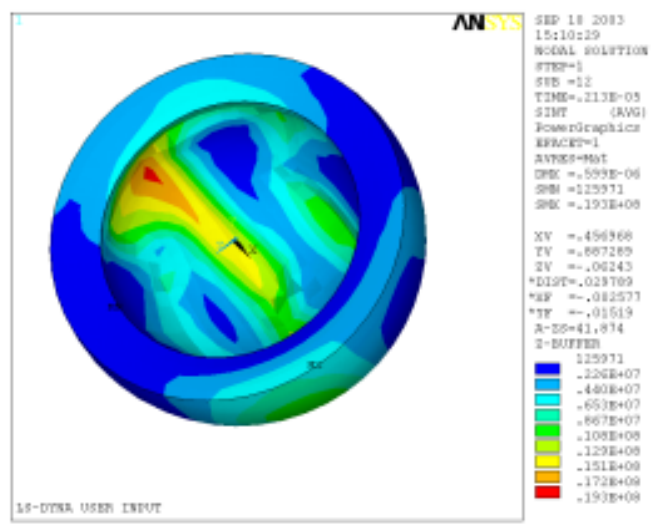

stresses 1

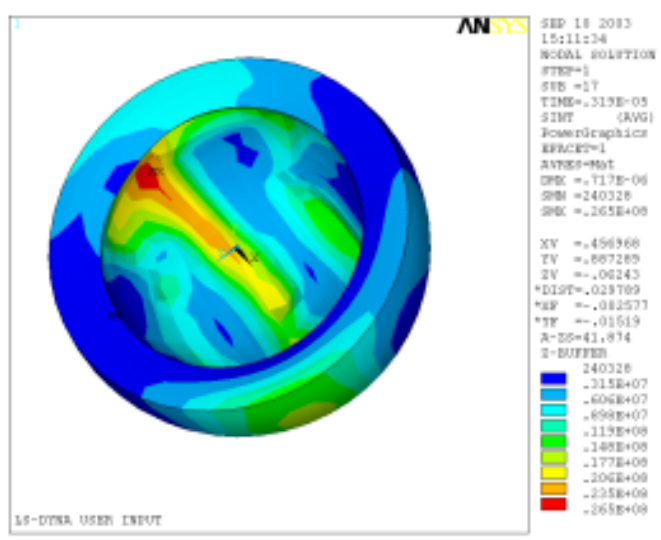

stresses 2

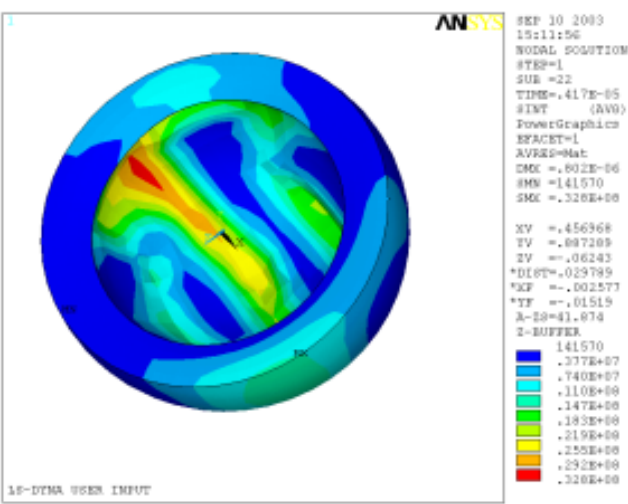

stresses 3

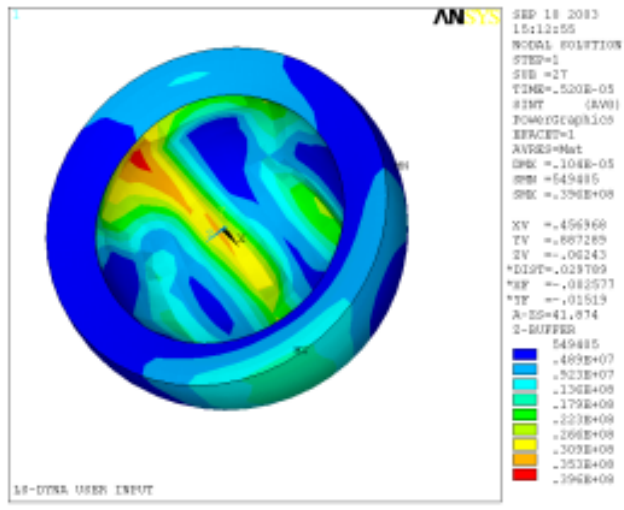

stresses 4

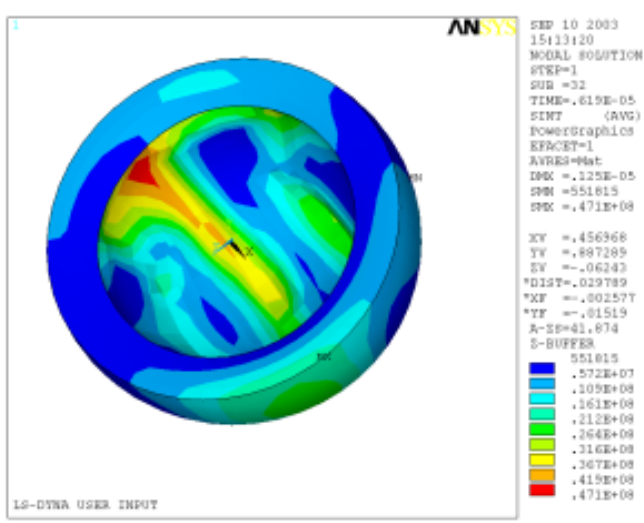

stresses 5

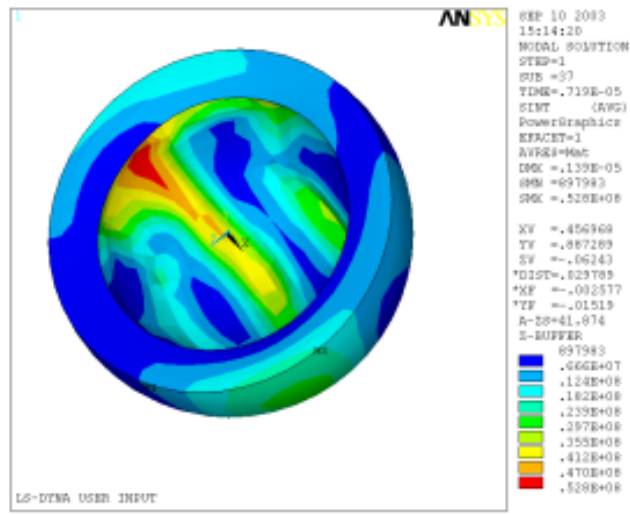

stresses 6

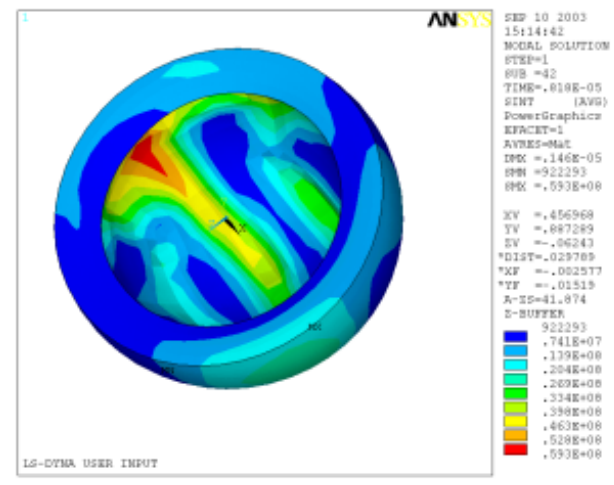

stresses 7

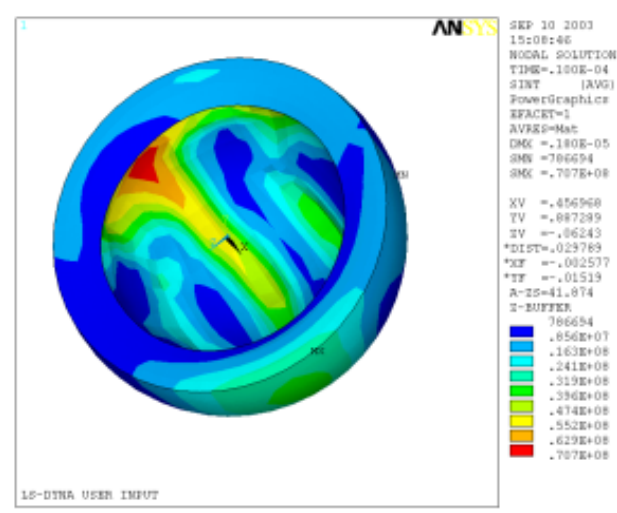

stresses 8

Figure 16. Maximal tangential stresses (in the acetabular cup) for eight different moments of the movement 


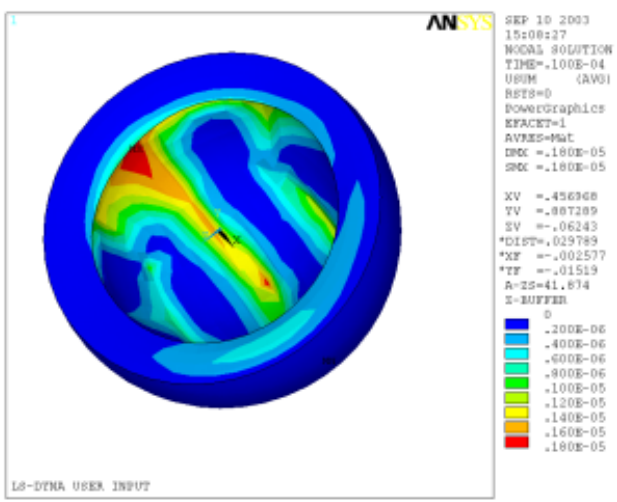

displacements 1

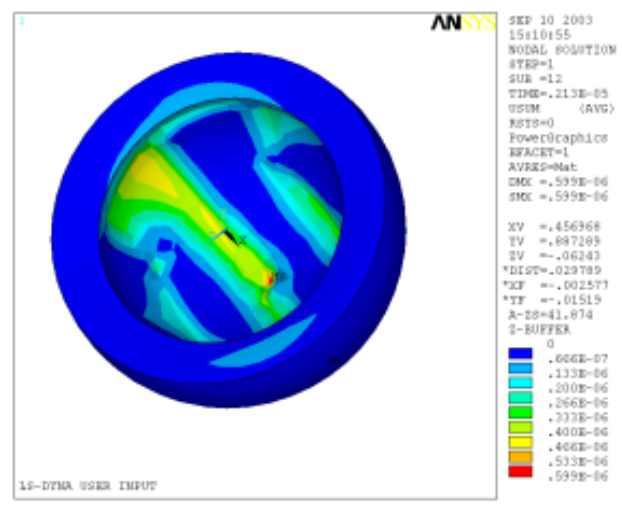

displacements 2

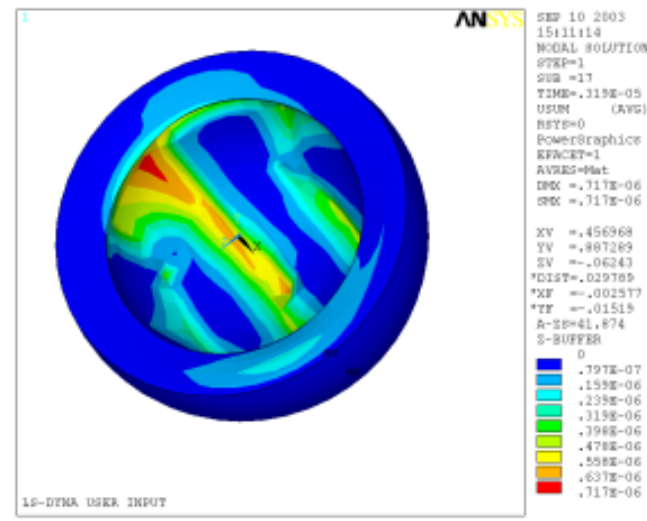

displacements 3

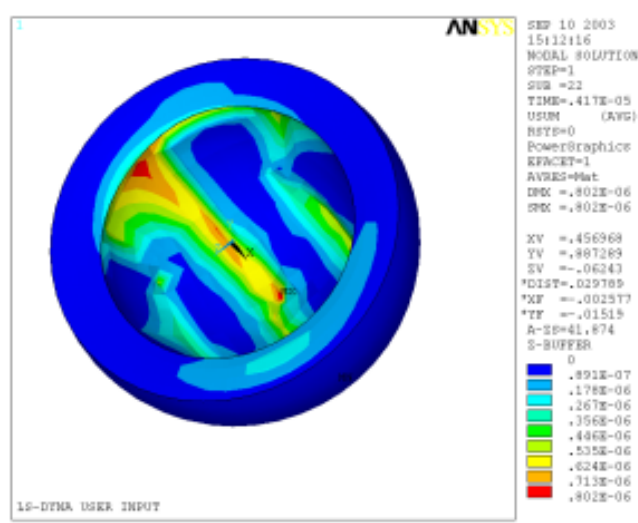

displacements 4
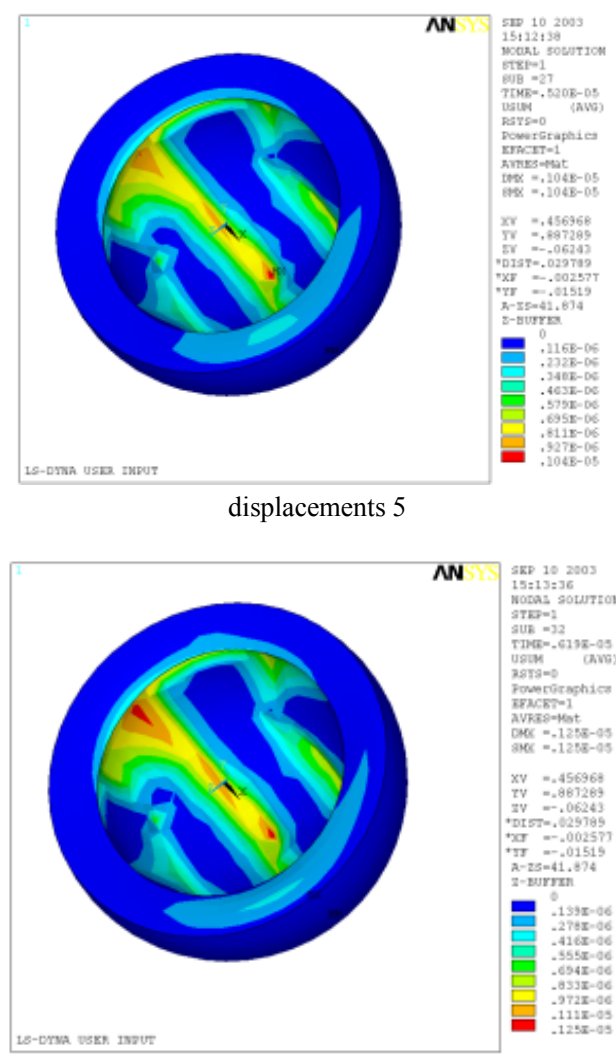

displacements 6

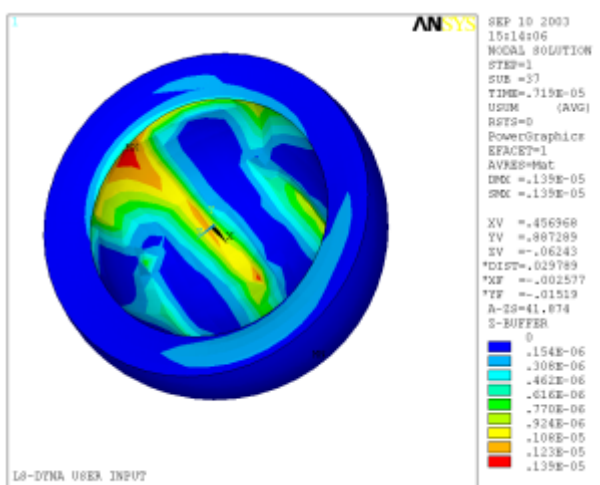

displacements 7

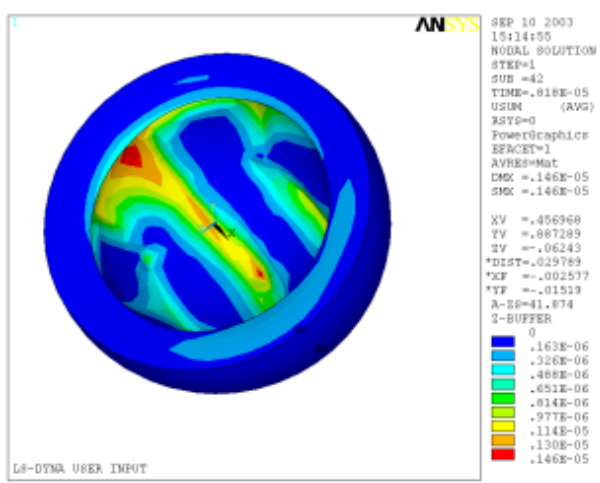

displacements 8

Figure 17. Normal displacements in the acetabular cup of a McKee-Farrar type prosthesis 
The femoral head was considered rigid and the acetabular cup - elastic isotropic.

A number of 3616 elements and 4845 nodes were taken into consideration. The time considered for the analysis was of $0,8 \times 10^{-3} \mathrm{sec}$., and the value of the sliding friction coefficient was 0,09 according to Tab.4.

Analyzing the recordings of the stresses and displacements of this prosthesis, a maximum normal displacement is found, approximately 2.2 times smaller than in the case of the prosthesis with self-directed movement balls.

The decrease of displacements is manifested based on the growth of almost 3.5 times of the normal tensions. This assessment has a justification in the fact that the real contact area at a classical type articulation is much bigger than at an articulation with balls.

Fig. 18 shows the evolution of the energies at the McKee type thimble having the acetabular cup made out of Ti6Al4V-ELI alloy and the thighbone head out of Co-Cr-Mo.

For a more complete general approach, an analysis of the efforts and of the movements appeared in the classical Stelit 21/UHMWPE prosthesis was also done. The time considered for the analysis was also of $0.8 \times 10^{-3} \mathrm{sec}$.

The following mechanical characteristics were also taken into account:

- for the thighbone head: $E=2.1 \times 10^{11} \mathrm{~N} / \mathrm{m}^{2} ; \rho=7850 \mathrm{~kg}$ $/ \mathrm{m}^{3}$ and $v=0.3$

- for the acetabular cup: $E=0.899 \mathrm{~N} / \mathrm{m}^{2} ; \rho=930 \mathrm{~kg} / \mathrm{m}^{3}$ and $v=0.36$.

For a more complete general approach, an analysis of the efforts and of the movements appeared in the classical Stelit 21/UHMWPE prosthesis was also done. The time considered for the analysis was also of $0.8 \times 10^{-3} \mathrm{sec}$.

The following mechanical characteristics were also taken into account:

- for the thighbone head: $E=2.1 \times 10^{11} \mathrm{~N} / \mathrm{m}^{2} ; \rho=7850 \mathrm{~kg}$ $/ \mathrm{m}^{3}$ and $v=0.3$

- for the acetabular cup: $E=0.899 \mathrm{~N} / \mathrm{m}^{2} ; \rho=930 \mathrm{~kg} / \mathrm{m}^{3}$ and $v=0.36$.

According to the experimental measurements presented in Tab. 4, the value of the friction coefficient introduced in the program, was: $\mu=0.065$.

The same values of the force that operates on the $y$ distance were considered, meaning $3200 \mathrm{~N}$, and of the rotation moment around the $z$ axis: $44.8 \mathrm{Nm}$.

Distortion was considered as being in the elastic field. The energies' variation curves of the classical hip prosthesis are presented in Fig. 17.

Figs. 19 and 20 show the maximum tangent efforts and the normal movements resulted from the analysis through the finite element method, for a few sequences of time.

Comparative analysis of the energy diagrams clearly shows a lower energy consumption $(2.115 \mathrm{Nm})$ at the prosthesis with self-directed movement spheres as opposed to $2 ., 22 \mathrm{Nm}$ at the McKee-Farrar type prosthesis, but much bigger than the classical prosthesis $(0,009 \mathrm{Nm})$.

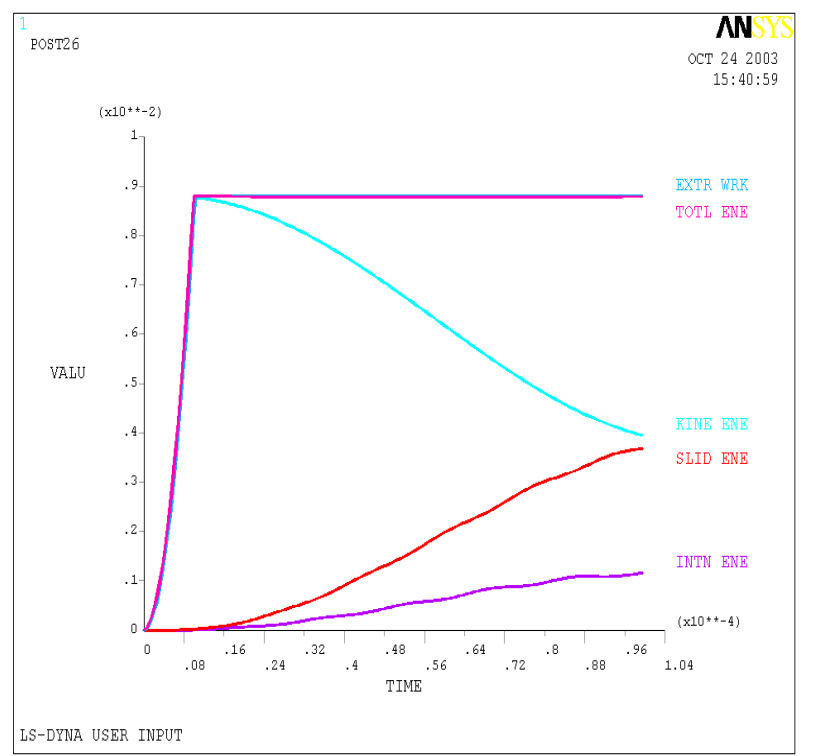

Figure 18. Energy variation diagrams of the classical Stelit $21 /$ UHMWPE prosthesis[9]

Comparative analysis of the energy diagrams clearly shows a lower energy consumption $(2.115 \mathrm{Nm})$ at the prosthesis with self-directed movement spheres as opposed to $2 ., 22 \mathrm{Nm}$ at the McKee-Farrar type prosthesis, but much bigger than the classical prosthesis $(0,009 \mathrm{Nm})$.
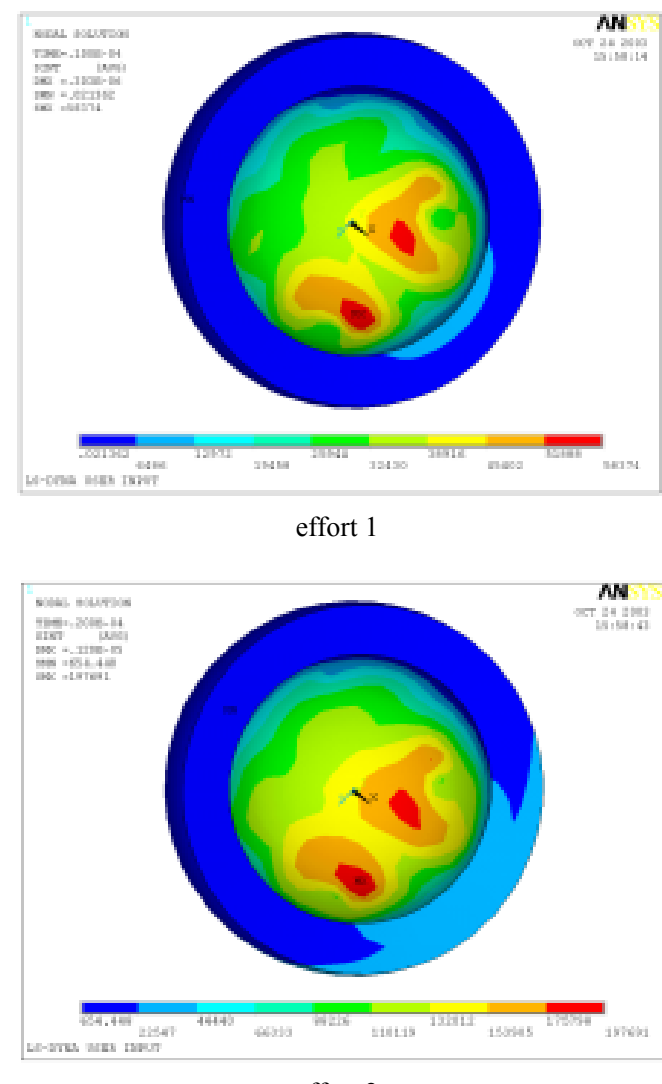

effort 2 


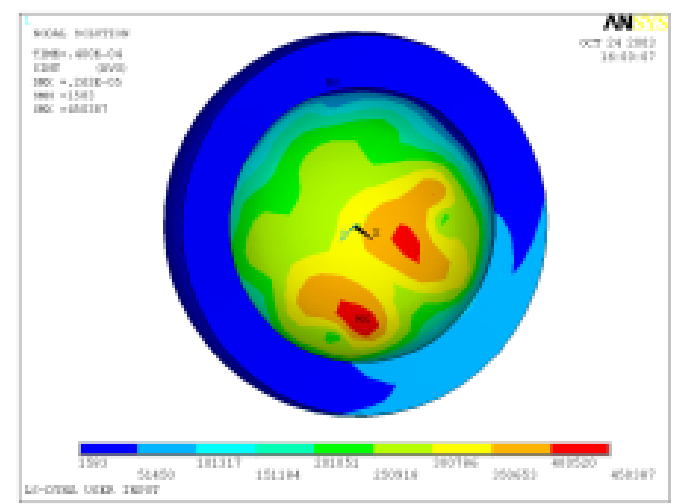

effort 4
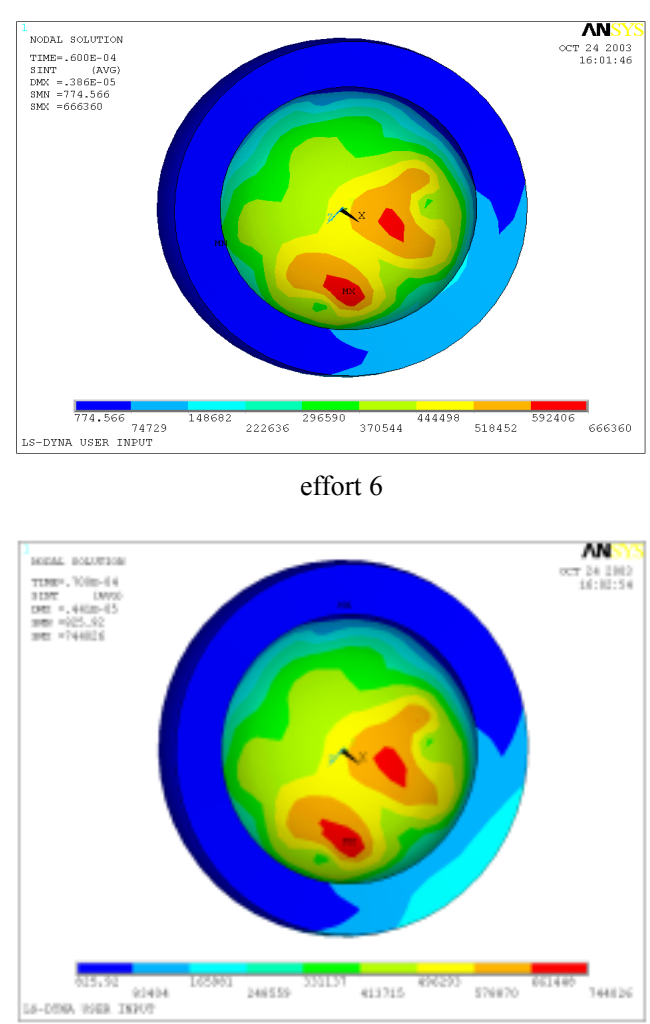

effort 7

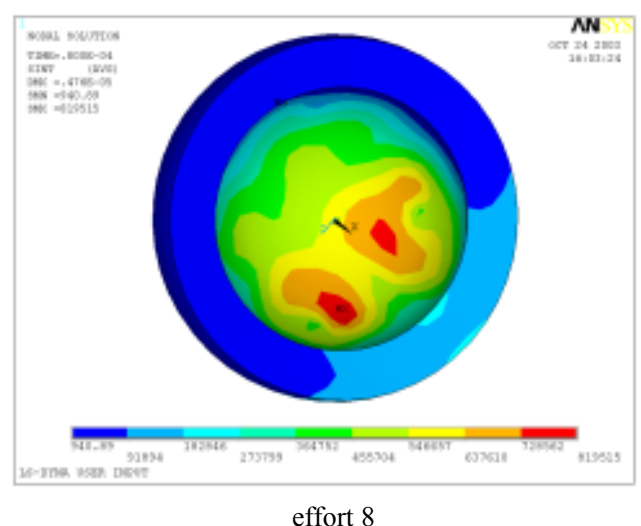

effort 8

Figure 19. Maximum tangent efforts in the acetabular cup of a Stelit 21/ UHMWPE prosthesis

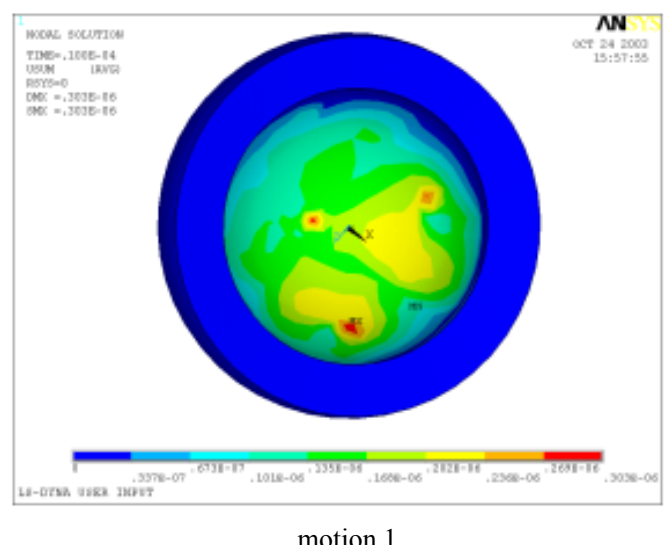

motion 1

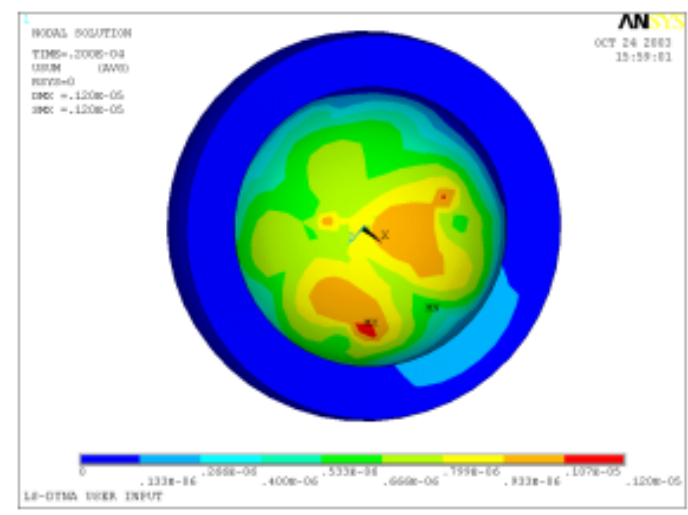

motion 2

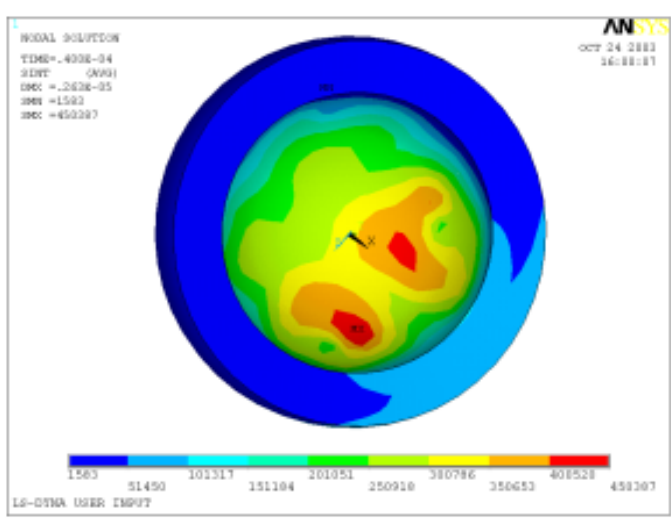

motion 4

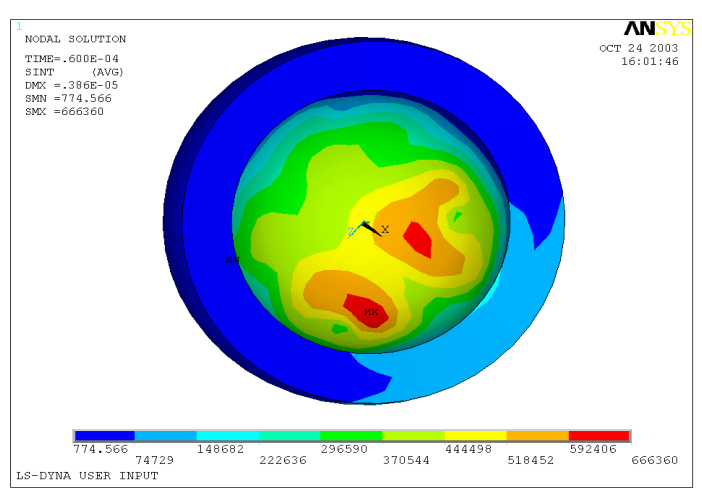

motion 6 


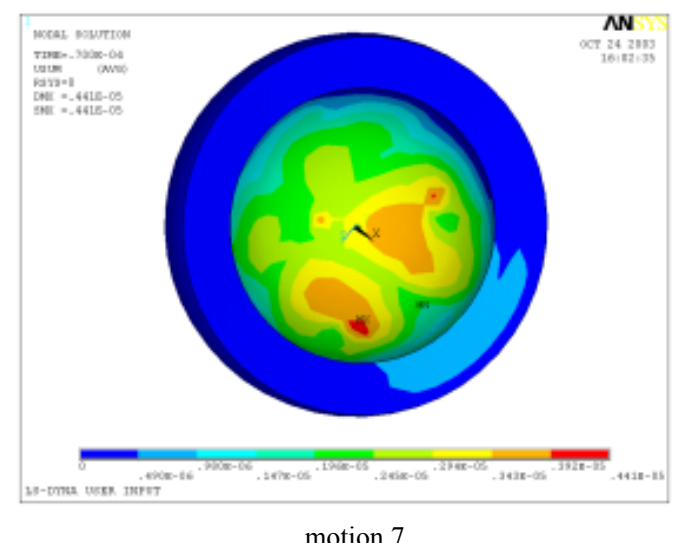

motion 7

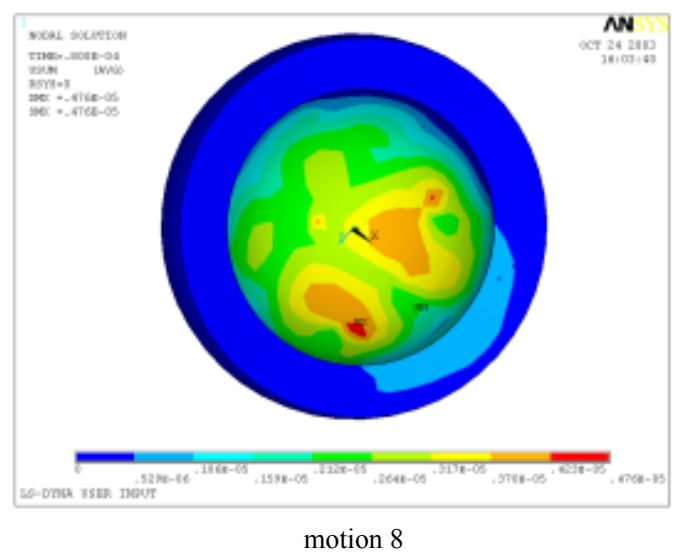

Figure 20. Normal movements in the acetabular cup of a classical Stelit 21/UHMWPE type prosthesis

This is a result of the chosen mathematical model that, due to the very short analysis time $\left(0.8 \times 10^{-3} \mathrm{sec}\right.$. $)$, could not offer information for more than the first movement sequence of the articulation. Subsequent research and studies will approach this aspect in the perspective of a new revolutionary constructive solution for orthopedic hip prosthesis

What have been presented so far are efforts of the Romanian scientific research for increasing the performances of orthopedic hip prosthesis. The results are partial, considering the fact that the studies are still in progress.

Are ongoing AMTI Boston hip simulator studies with physiological loading and motion, to determining the optimal materials and thin films coatings on acetabular cup. Also, researches are ongoing to optimize the lubrication regime. The results will be presented as they become conclusive and validated.

\section{ACKNOWLEDGEMENTS}

The authors would like to thank the Romanian Academy for its material and technical support offered in order to achieve these researches.

\section{REFERENCES}

[1] Sadeghi-Mehr, M., "Investigations of Rolling Element Bearing for Hip Joint Prosthesis", PhD Thesis, Imperial College of Science, Technology and Medicine University, London, 1997.

[2] Katsutashi B., S. Kiyoshi, US Patent 5092898 / 03.03.1992.

[3] Davant, J.P., "Chirurgie de la hance. Mieux vivre avec une prosthesis”, Fonder l'avenir, 23, pp. 53-56, 1995.

[4] Capitanu L., Florescu V. About some tribological aspects of total hip prostesis. A possible technical solution in improvement tribological characteristics (in Romanian), $7^{\text {th }}$ International Conference on Tribology, ROTRIB'96, pp. 366-373.

[5] Wang A,.Essner A., Polineni V.K., Stark C., Dumbleton J.H. Lugrication and wear of ultra-high molecular weight polyethylene in total joint replacements. Tribology International (1998) 1-3,1067-1073.

[6] Wang A,.Sun D.C., Stark C., Dumbleton J.H. Wear mechanisms of UHMWPE in total joints replacements. Wear 181-183 (1995), 241-249.

[7] Capitanu L, Florescu V., Petrescu F. Experimental Studies of Hip Replacement, The Annual Symposium of the Institute of Solid Mechanics, SISOM 2001, Bucharest, pp.271-274.

[8] Iarovici, A., Capitanu, L., Florescu, V., Baubec, M., "Hip Joint Prosthesis with rolling bodies", Proceedings of the Romanian Academy - Series A: Mathematics, Physics, Technical Sciences, Information Science, 1, 1-2, pp. 37-44, S. 2001.

[9] Iarovici, A., Capitanu, L., Florescu, V., Baubec, M., Petrescu, F., "Hip Joint Prosthesis with Rolling Bodies, Part I - The Balls Arrangement Analysis", Proceedings of the Annual Symposium of the Institute of Solid Mechanics - SISOM 2001, pp. 251-258.

[10] Iarovici, A., Capitanu, L., Florescu, V., Baubec, M., Petrescu, F., Hip Joint Prosthesis with Rolling Bodies, Part II Numerical and Graphycal Examples, Proceedings of the Annual Symposium of the Institute of Solid Mechanics SISOM 2001, pp. 259-263.

[11] Iarovici, A., Capitanu, L., Onisoru, J., Florescu, V., Baubec, M., Stresses and deformations analysis for Hip Joint Prostheses with rolling bodies, Proceedings of the Annual Symposium of the Institute of Solid Mechanics SISOM 2002, pp. 263-268. 